\title{
Dynamic Output Feedback Based Active Decentralized Fault-Tolerant Control for Reconfigurable Manipulator with Concurrent Failures
}

\author{
Yuanchun Li, ${ }^{1}$ Fan Zhou, ${ }^{1}$ and Bo Zhao ${ }^{1,2}$ \\ ${ }^{1}$ Department of Control Engineering, Changchun University of Technology, Changchun 130012, China \\ ${ }^{2}$ State Key Laboratory of Management and Control for Complex Systems, Institute of Automation, \\ Chinese Academy of Sciences, Beijing 100190, China
}

Correspondence should be addressed to Bo Zhao; zhaob09@mails.jlu.edu.cn

Received 10 July 2014; Revised 27 November 2014; Accepted 29 November 2014

Academic Editor: Gerhard-Wilhelm Weber

Copyright (C) 2015 Yuanchun Li et al. This is an open access article distributed under the Creative Commons Attribution License, which permits unrestricted use, distribution, and reproduction in any medium, provided the original work is properly cited.

\begin{abstract}
The goal of this paper is to describe an active decentralized fault-tolerant control (ADFTC) strategy based on dynamic output feedback for reconfigurable manipulators with concurrent actuator and sensor failures. Consider each joint module of the reconfigurable manipulator as a subsystem, and treat the fault as the unknown input of the subsystem. Firstly, by virtue of linear matrix inequality (LMI) technique, the decentralized proportional-integral observer (DPIO) is designed to estimate and compensate the sensor fault online; hereafter, the compensated system model could be derived. Then, the actuator fault is estimated similarly by another DPIO using LMI as well, and the sufficient condition of the existence of $H_{\infty}$ fault-tolerant controller in the dynamic output feedback is presented for the compensated system model. Furthermore, the dynamic output feedback controller is presented based on the estimation of actuator fault to realize active fault-tolerant control. Finally, two 3-DOF reconfigurable manipulators with different configurations are employed to verify the effectiveness of the proposed scheme in simulation. The main advantages of the proposed scheme lie in that it can handle the concurrent faults act on the actuator and sensor on the same joint module, as well as there is no requirement of fault detection and isolation process; moreover, it is more feasible to the modularity of the reconfigurable manipulator.
\end{abstract}

\section{Introduction}

The rapid development of robotics leads the reconfigurable manipulators to be variously applied to the potential unstructured environments, especially in the fields where human cannot intervene directly, such as the space station, nuclear power plant, and battle field. However, once the fault appeared in the system, it might deteriorate the performance or cause the loss of the system functionality, even stability. As a result, there is an increasing demand for safety, reliability, and performance of reconfigurable manipulator systems. Therefore, it is an urgent requirement to design control systems which can tolerate the occurrence of failures during the operation, in order to guarantee the stability and functionality and maintain the acceptable performance as well.
Generally speaking, two strategies, namely, the passive fault-tolerant control (PFTC) and the active fault-tolerant control (AFTC), were carried out to achieve the aim at FTC [1]. For the PFTC, the control structure and parameters have been redesigned to go against the occurring of failures. This means that the FTC was fixed to tolerate a certain set of faults without any change in the controller. Du et al. [2] obtained the fault information by estimating the outputs of the actuators and then compared them with the corresponding prescribed control inputs; hereafter, the FTC was developed by choosing a safe-park point. Jiang et al. [3] presented the sliding mode FTC method in the view of the nonlinear flexible spacecraft flywheel failure, while in fact it was difficult to obtain the minimum value of the spacecraft flywheel fault. Brambilla et al. [4] adopted an optimal second-order sliding mode control method to design observer-control law, by using 
the unknown input observer and generalized observer to analyze residuals, but this method can only detect a single component failure. In [5], a decentralized tuning PID output feedback controller was utilized to ensure the stability of large flexible space structures (LFSS) suffered sensor and actuator failures. Moreover, a common solution in the PFTC when some severe failures are taken into account does not always exist. In addition, it usually presented a low performance even though it exists. On the other hand, the AFTC may change the control structure and/or parameters to overcome the bad effect on the whole control systems aroused by the fault. Even when necessary, it needs to introduce a detection and estimation module to detect and estimate when the fault occurs. Hereafter, a supervisory controller should be reconstructed based on the estimated information in the case of the occurrence of severe faults $[6,7]$, such that it can guarantee the faulty system's stability and provide acceptable control performance. In [8-12], only single fault is handled with AFTC, but the concurrent failures on actuator and sensor always occur in actual fact. In this regard, Rotondo et al. [13] used virtual actuator and sensor to correct the actual actuator and sensor faults, which achieved the objective of FTC based on the dynamical controller reconfiguration. Sami and Patton [14] proposed a new architecture based on a combination of actuator and sensor Takagi-Sugeno (T-S) proportional state estimators augmented with proportional and integral feedback (PPI) fault estimators, together with a T-S dynamic output feedback control for time-varying reference tracking.

In recent literatures, some effort has been made for the reconfigurable manipulators in fault. Yuan et al. [15] introduced an energy efficiency monitor approach to detect the fault, where the operation failure was reflected by the efficiency decline of mechanical system. The measurement of each joint torque is used not only to control the running state, but also to reflect the output capacity. The method based on torque measurement is independent of the whole dynamic model of robot systems. Ahmad et al. [16] presented a distributed fault detection method, which can gain the forecast error through comparing the joint torque signal and torque estimation being filtered. Zhao and Li [17] were concerned with the active fault-tolerant control problem for reconfigurable manipulator actuator based on local joint information. This scheme processed a simple control structure, as well as the fault could be isolated and tolerated in subsystem, and it can be easily applied to different configurations without any parameters modification. However, only a single fault in the actuator or sensor was taken into account to be handled in the aforementioned methods, which limited the availability in practice.

There are several methods successfully used in controlling reconfigurable manipulator. In centralized control approach, Li et al. [18] utilized the elastic parameters of joint module, which were identified by fuzzy logic, to build finite element model of reconfigurable robot; then, based on the BP neural network and genetic algorithm, the vibration control method was proposed based on the finite element model. Sun et al. [19] divided 4-DOF module into posture coupled subsystems and position feedback subsystem and simultaneously decomposed workspace into the above two subspaces to solve the inverse position problem through forecasting method. Biglarbegian et al. [20] presented Type-2 TSK fuzzy logic control method aimed at the reconfigurable manipulators with uncertain dynamic parameters. This control structure had a complex and fixed control structure and lacked flexibility; thus, it was difficult to be implemented to the reconfigurable manipulator when its configuration changed. The other one was distributed control method. Muller et al. [21] simplified the hardware of control system to ensure the flexibility of system reconstruction and coordinated to operate all modular robots through independent central control. Zhu and Lamarche [22] described the system as a set of subsystems through virtual decomposition and then used the exchange information among modules to design the subsystem's controller. The distributed control method can reduce computational complexity and has a more harmonious and flexible structure compared to those in centralized control; it makes the system compatibility not only better, but also more suitable to the concept of modularization. This distributed control could conduct more thorough coordinate control; however, the time delay in communication can result in imprecise control performance. To reduce the difficulty in controller design, the decentralized control strategy is developed in a large-scale system. In fact, the main property of the reconfigurable manipulator system lies in different configurations and different degree of freedom. Therefore, it is more suitable to take a joint module as a subsystem, and the decentralized control method can satisfy its main property. Kirchoff and Melek [23] designed a PID robust controller based on independent joint information for industrial robot. Li [24] introduced a dispersion saturated type of robust control method only considering the single joint dynamics after the system was decoupled and treated the influence of other joints' dynamics as external disturbance. The controller design in decentralized control approach utilizes only local information; thus, it is more suitable for the system with an uncertain degree of freedom and different configurations.

This paper tries to address an ADFTC for reconfigurable manipulator with concurrent failures. This idea focuses on the observer design for isolating and estimating the actuator and sensor faults for the purpose of fault compensation. It decomposes the entire system into a set of interconnected subsystems for developing decentralized control architecture. A DPIO is designed through using LMI technique to estimate and compensate the sensor fault online, and the compensated system model is derived. Similarly, another DPIO is established with the sufficient condition of the existence of $H_{\infty}$ fault-tolerant controller and presented in the presence of the dynamic output feedback. Simultaneously, the ADFTC is realized by the estimation of the faults based on the dynamic output feedback. Finally, simulation results show the stability and accuracy in the tracking system with simultaneously acting actuators and sensors faults.

The main advantages of the proposed approach lie in the following: (i) Only local information is used to design the ADFTC for reconfigurable manipulator with the concept of decentralized control, which can tolerate the concurrent faults acting in actuator and sensor in an independent joint 
module. (ii) LMI technique is used in the design procedure of DPIOs and dynamic output feedback controller, simplifying the control structure and making the proof process of system stability easier on the condition of ensuring the system stability. (iii) There is no requirement of FDI unit here, so it saves the reconfiguration time, which is necessary in the conventional AFTC. (iv) Compared to the existing results, the dynamic output feedback is utilized as the state feedback in the proposed scheme; meanwhile, it could balance the contradictions between the irreplaceable state feedback and the difficulty in physical realization.

This paper is presented in the following order. Section 2 describes nonlinear interconnected subsystem dynamic model of the reconfigurable manipulator, including the systems with fault or without fault. Section 3 enters into a description of the observers followed by two subsections, which illustrate the stability and performance design conditions for (i) sensor fault estimate observer and (ii) the actuator fault estimate observer. In Section 4, the dynamic output feedback controller is designed and it illustrates the stability and performance design conditions. In Section 5, the effectiveness of the proposed ADFTC method is verified by the simulation results of two 3-DOF reconfigurable manipulators with different configurations. Some conclusions are drawn in Section 6.

\section{Problem Description}

For the development of decentralized control, consider the entire reconfigurable manipulator with $n$-DOF as a set of nonlinear interconnected subsystems, which are composed of a general joint module. And the subsystem $I$ in the reconfigurable manipulator system can be presented by the following state equation [25]:

$$
S_{i}:\left\{\begin{array}{l}
\dot{x}_{i}=A_{i} x_{i}+B_{i}\left[f_{i}\left(q_{i}, \dot{q}_{i}\right)+g_{i}\left(q_{i}\right) u_{i}+h_{i}(q, \dot{q}, \ddot{q})\right] \\
y_{i}=C_{i} x_{i},
\end{array}\right.
$$

where $x_{i}=\left[x_{i 1}, x_{i 2}\right]^{T}=\left[q_{i}, \dot{q}_{i}\right]^{T}(i=1,2, \ldots, n)$ is the state vector of the subsystem $S_{i}$ and $y_{i}$ is the output of the subsystem $S_{i}$. The matrices

$$
\begin{gathered}
A_{i}=\left[\begin{array}{ll}
0 & 1 \\
0 & 0
\end{array}\right], \quad B_{i}=\left[\begin{array}{l}
0 \\
1
\end{array}\right], \quad C_{i}=\left[\begin{array}{ll}
1 & 0 \\
0 & 1
\end{array}\right], \\
f_{i}\left(q_{i}, \dot{q}_{i}\right)=M_{i}^{-1}\left(q_{i}\right)\left[-C_{i}\left(q_{i}, \dot{q}_{i}\right) \dot{q}_{i}-G_{i}\left(q_{i}\right)\right], \\
g_{i}\left(q_{i}\right)=M_{i}^{-1}\left(q_{i}\right), \\
h_{i}(q, \dot{q}, \ddot{q})=-M_{i}^{-1}\left(q_{i}\right) Z_{i}(q, \dot{q}, \ddot{q}) .
\end{gathered}
$$

For the subsystem suffering actuator and sensor failures concurrently, the faulty dynamic model can be expressed as

$$
S_{i f}:\left\{\begin{aligned}
& \dot{x}_{i}=A_{i} x_{i}+B_{i}[ {\left[g_{i}\left(q_{i}\right)\left(u_{i}+f_{i a}\right)\right.} \\
&\left.+f_{i}\left(q_{i}, \dot{q}_{i}\right)+h_{i}(q, \dot{q}, \ddot{q})\right] \\
& y_{i}=C_{i} x_{i}+D_{i} f_{i s}
\end{aligned}\right.
$$

here, $f_{i a}\left(q_{i}, \dot{q}_{i}, \dot{u}_{i}\right)$ denotes the actuator fault function, $D_{i}=$ $\left[\begin{array}{ll}1 & 0 \\ 0 & 1\end{array}\right]$ is the sensor fault distribution matrix, and $f_{i s}=$ $\left[\begin{array}{ll}f_{\text {is } 1} & f_{\text {is } 2}\end{array}\right]^{T}$ satisfies $\left\|f_{\text {is }}\right\| \leq \rho_{i}(t)$, where $\rho_{i}(t)$ is a continuous positive-definite function.

The control objective is to design an active decentralized fault-tolerant controller in order to guarantee the whole closed-loop system stability in the case of the system suffering concurrent actuator and sensor faults. In other words, the proposed fault-tolerant control scheme should make the outputs of the entire system follow the desired trajectories even though concurrent faults occur.

\section{Decentralized Proportional-Integral Observer Design}

3.1. Sensor Fault Observer Design. In this subsection, a decentralized proportional-integral observer is designed for the faulty dynamic model (3) in order to estimate the sensor fault.

Assumption 1. The desired trajectories $q_{i}^{d}, \dot{q}_{i}^{d}$, and $\ddot{q}_{i}^{d}$ are bounded.

Assumption 2. The subsystem actuator fault function $f_{i a}\left(q_{i}, \dot{q}_{i}, \dot{u}_{i}\right)$ and the sensor fault function $f_{i s}$ are bounded as $\left\|f_{i a}\right\| \leq f_{i a}^{*}$ and $\left\|f_{i s 1}\right\| \leq f_{i s 1}^{*}$.

Introduce a first order filter as [26]

$$
\dot{z}_{i}=-A_{i a} z_{i}+B_{i a} y_{i}
$$

Next, putting the output of (3) into (4), we have

$$
\dot{z}_{i}=-A_{i a} z_{i}+B_{i a} C_{i} x_{i}+B_{i a} D_{i} f_{i s}
$$

where $z_{i}=\left[\begin{array}{ll}z_{i 1} & z_{i 2}\end{array}\right]^{T}, y_{i}$ is the joint position sensor and velocity sensor output signal, and $-A_{i a} \in R^{2 \times 2}$ is a Hurwitz matrix with $\operatorname{det}\left(B_{i a}\right) \neq 0$.

Combining the states of (3) and (5), it is gained that

$$
\bar{S}_{i f}:\left\{\begin{array}{c}
\dot{\bar{x}}_{i}=\bar{A}_{i} \bar{x}_{i}+\bar{B}_{i}\left[f_{i}\left(q_{i}, \dot{q}_{i}\right)+g_{i}\left(q_{i}\right)\left(u_{i}+f_{i a}\right)\right. \\
\left.+h_{i}(q, \dot{q}, \ddot{q})\right]+\bar{D}_{i} f_{i s}, \\
\bar{y}_{i}=\bar{C}_{i} \bar{x}_{i},
\end{array}\right.
$$

where $\bar{x}_{i}=\left[\begin{array}{c}x_{i} \\ z_{i}\end{array}\right], \bar{A}_{i}=\left[\begin{array}{cc}A_{i} & 0 \\ B_{i a} C_{i}-A_{i a}\end{array}\right], \bar{B}_{i}=\left[\begin{array}{c}B_{i} \\ 0\end{array}\right], \bar{C}_{i}=\left[\begin{array}{ll}0 & I\end{array}\right]$, and $\bar{D}_{i}=\left[\begin{array}{c}0 \\ B_{i a} D_{i}\end{array}\right]$.

Now, utilize the RBF neural networks to approximate the unknown term $f_{i}\left(q_{i}, \dot{q}_{i}\right)$ and uncertainty term $g_{i}\left(q_{i}\right)$ as follows:

$$
\begin{gathered}
f_{i}\left(q_{i}, \dot{q}_{i}, W_{i f}\right)=W_{i f}^{T} \Phi_{i f}\left(q_{i}, \dot{q}_{i}\right)+\varepsilon_{i f}, \\
g_{i}\left(q_{i}, W_{i g}\right)=W_{i g}^{T} \Phi_{i g}\left(q_{i}\right)+\varepsilon_{i g},
\end{gathered}
$$

where $W_{i f}$ and $W_{i g}$ are the ideal neural network weights, respectively. $\Phi(\cdot)$ is the neural network basis function, and $\varepsilon_{i f}$ and $\varepsilon_{i g}$ are the neural network approximation errors, respectively. 
Define $\widehat{W}_{i f}$ and $\widehat{W}_{i g}$ as the estimations of $W_{i f}$ and $W_{i g} \cdot \widehat{f}_{i}\left(q_{i}, \dot{q}_{i}, \widehat{W}_{i f}\right)$ and $\widehat{g}_{i}\left(q_{i}, \widehat{W}_{i g}\right)$ expressed as (8) are the estimations of $f_{i}\left(q_{i}, \dot{q}_{i}, W_{i f}\right)$ and $g_{i}\left(q_{i}, W_{i g}\right)$, respectively:

$$
\begin{aligned}
\widehat{f}_{i}\left(q_{i}, \dot{q}_{i}, \widehat{W}_{i f}^{T}\right) & =\widehat{W}_{i f}^{T} \widehat{\Phi}_{i f}\left(q_{i}, \dot{q}_{i}\right), \\
\widehat{g}_{i}\left(q_{i}, \widehat{W}_{i g}^{T}\right) & =\widehat{W}_{i g}^{T} \widehat{\Phi}_{i g}\left(q_{i}\right),
\end{aligned}
$$

where the adjustable parameters are updated by the following adaptive laws:

$$
\begin{gathered}
\dot{\widehat{W}}_{i f}=\eta_{i f} e_{i}^{T} P_{i} \bar{B}_{i} \widehat{\Phi}_{i f}\left(q_{i}, \dot{q}_{i}\right), \\
\dot{\bar{W}}_{i g}=\eta_{i g} e_{i}^{T} P_{i} \bar{B}_{i} \widehat{\Phi}_{i g}\left(q_{i}\right) u_{i},
\end{gathered}
$$

where $\eta_{i f}$ and $\eta_{i g}$ are positive constants.

Note that a challenge in implementing the decentralized control is to compensate the coupling torque caused by the interconnected joint modules. In such a scenario, the following assumption is presented.

Assumption 3. The interconnection term $h_{i}(q, \dot{q}, \ddot{q})$ is bounded by [25]

$$
\left|h_{i}(q, \dot{q}, \ddot{q})\right| \leq \sum_{j=1}^{n} d_{i j} E_{j},
$$

with $d_{i j} \geq 0$ and $E_{j}=1+\left\|e_{i}^{T} P_{i} \bar{B}_{i}\right\|+\left\|e_{i}^{T} P_{i} \bar{B}_{i}\right\|^{2}$.

Similarly, another RBF neural network term $\widehat{R}_{i}\left(\left\|e_{i}^{T} P_{i} \bar{B}_{i}\right\|\right.$, $\left.\widehat{W}_{i p}\right)$ is introduced to compensate the effect of interconnection term and defined as follows:

$$
v_{i}(t)=-\operatorname{sgn}\left(e_{i}^{T} P_{i} \bar{B}_{i}\right) \widehat{R}_{i}\left(\left\|e_{i}^{T} P_{i} \bar{B}_{i}\right\|, \widehat{W}_{i p}\right) .
$$

Similarly, another RBF neural network expressed as (12) is proposed to achieve this goal:

$$
\widehat{R}_{i}\left(\left\|e_{i}^{T} P_{i} \bar{B}_{i}\right\|, \widehat{W}_{i p}\right)=\widehat{W}_{i p}^{T} \Phi_{i p}\left(\left\|e_{i}^{T} P_{i} \bar{B}_{i}\right\|\right),
$$

where $W_{i p}$ is the ideal neural network weight and $\Phi_{i p}(\cdot)$ is the neural network basis function. $\widehat{W}_{i p}$ and $\widehat{\Phi}_{i p}$ are the estimations of $W_{i p}$ and $\Phi_{i p}$, respectively. $\widetilde{W}_{i p}=\widehat{W}_{i p}-W_{i p}$ and $\widetilde{\Phi}_{i p}=\widehat{\Phi}_{i p}-\Phi_{i p}$ are relative estimation errors. And $\widehat{W}_{i p}$ can be updated by

$$
\dot{\widehat{W}}_{i p}=\eta_{i p}\left\|e_{i}^{T} P_{i} \bar{B}_{i}\right\| \widehat{\Phi}_{i p} ;
$$

also, $\eta_{i p}$ is a positive constant.

Finally, define approximation error:

$$
\begin{gathered}
\omega_{i 1}=W_{i f}^{T} \widetilde{\Phi}_{i f}\left(q_{i}, \dot{q}_{i}\right)+W_{i g}^{T} \widetilde{\Phi}_{i g}\left(q_{i}\right) u_{i}+\varepsilon_{i f}+\varepsilon_{i g} u_{i}, \\
\omega_{i 2}=R_{i}\left\|e_{i}^{T} P_{i} \bar{B}_{i}\right\|-\widehat{W}_{i p}^{T} \widehat{\Phi}_{i p}\left(\left\|e_{i}^{T} P_{i} \bar{B}_{i}\right\|\right), \\
\omega_{i}=\left|\omega_{i 1}\right|+\left|\omega_{i 2}\right|,
\end{gathered}
$$

where $R_{i}\left(\left\|e_{i}^{T} P_{i} \bar{B}_{i}\right\|\right)=n \max _{i j}\left\{d_{i j}\right\} E_{i}$.
Next, the decentralized proportional-integral observer (DPIO) as (15) is proposed to simultaneously estimate the system states and sensor fault [27]:

$$
\hat{\bar{S}}_{i f}:\left\{\begin{array}{c}
\dot{\overline{\hat{x}}}_{i}=\bar{A}_{i} \hat{\bar{x}}_{i}+\bar{B}_{i}\left[\widehat{f}_{i}\left(\widehat{q}_{i}, \dot{\bar{q}}_{i}\right)+\widehat{g}_{i}\left(\widehat{q}_{i}\right)\left(u_{i}+f_{i a}\right)+v_{i}\right. \\
\left.\quad-\beta_{i} \operatorname{sgn}\left(e_{i}^{T} P_{i} \bar{B}_{i}\right)\right] \\
\quad+\bar{D}_{i} \widehat{f}_{i s}+K_{i p}\left(\bar{y}_{i}-\widehat{\bar{y}}_{i}\right) \\
\hat{\bar{y}}_{i}=\bar{C}_{i} \widehat{\bar{x}}_{i} \\
\widehat{f}_{i s}=K_{i v} \bar{C}_{i} \dot{e}_{i x}+K_{i l} \bar{C}_{i} e_{i x},
\end{array}\right.
$$

where $\beta_{i} \operatorname{sgn}\left(e_{i}^{T} P_{i} \bar{B}_{i}\right)$ is a robust term, which is utilized to go against the effects of neural network approximation error on the observer.

Now define the state estimation errors as $e_{i x}=\bar{x}_{i}-\hat{\bar{x}}_{i}$ and sensor fault estimation errors as $e_{f i s}=f_{i s}-\widehat{f}_{i s}$, where $\hat{\bar{x}}_{i}$ is the estimation of the state vector $\bar{x}_{i}$ and $\widehat{f}_{i s}$ is the estimation of the sensor fault $f_{i s}$.

Combining (6) and (15), the error dynamics are as follows:

$$
\begin{aligned}
\dot{e}_{i x}= & \dot{\bar{x}}_{i}-\dot{\overline{\bar{x}}}_{i}=\left(\bar{A}_{i}-K_{p s} \bar{C}_{i}\right) e_{i}+\bar{D}_{i} e_{f i s}+\bar{B}_{i} m_{s}, \\
\dot{e}_{f i s}= & f_{i l}-\widehat{f}_{i l} \\
= & f_{i l}-\left[K_{i l} \bar{C}_{i}+K_{i v} \bar{C}_{i}\left(\bar{A}_{i}-K_{i p} \bar{C}_{i}\right)\right] e_{i x} \\
& +K_{i v} \bar{C}_{i} \bar{D}_{i} e_{f i s}+K_{i v} \bar{C}_{i} \bar{B}_{i} m_{s},
\end{aligned}
$$

where

$$
\begin{gathered}
m_{s}=\left[\left(f_{i}-\widehat{f}_{i}\right)+\left(g_{i}-\widehat{g}_{i}\right)\left(u_{i}+f_{i a}\right)\right. \\
\left.-v_{i}+h_{i}+\beta_{i} \operatorname{sgn}\left(e_{i}^{T} P_{i} \bar{B}_{i}\right)\right] .
\end{gathered}
$$

Note that the augmented estimator will then be of the following form:

$$
\dot{e}_{i s}=A_{i s} e_{i s}+N_{i s} z_{i s},
$$

where $e_{i s}=\left[\begin{array}{c}e_{i x} \\ e_{\text {fis }}\end{array}\right]$ and

$$
\begin{gathered}
A_{i s}=\left[\begin{array}{cc}
\bar{A}_{i}-K_{p s} \bar{C}_{i} & \bar{D}_{i} \\
K_{i l} \bar{C}_{i}+K_{i v} \bar{C}_{i}\left(\bar{A}_{i}-K_{i p} \bar{C}_{i}\right) & K_{i v} \bar{C}_{i} \bar{D}_{i}
\end{array}\right], \\
N_{i s}=\left[\begin{array}{cc}
\bar{B}_{i} & 0 \\
K_{i v} \bar{C}_{i} \bar{B}_{i} & I
\end{array}\right], \quad z_{i s}=\left[\begin{array}{c}
m_{s} \\
\dot{f}_{i s}
\end{array}\right] .
\end{gathered}
$$

Lemma 4 (see [28]). In the given system, the eigenvalues of the system are located in a LMI region in the complex plane defined by $D(q, r)$ which is defined by merging different eigenvalues constraints to produce a $D(q, r)$ LMI region in which $q$ and $r$ are the radius and center of the disc region. If there exist symmetric 
positive-definite matrices $P$ and $Q$ and matrices $K_{i p}, K_{i l}$, and $K_{i v}$, as well as the corresponding LMI such that

$$
\left[\begin{array}{cccc}
A_{11} & A_{12} & P \bar{B}_{i} & 0 \\
A_{12}^{T} & A_{22} & K_{i v} \bar{C}_{i} \bar{B}_{i} & I \\
P \bar{B}_{i} & K_{i v} \bar{C}_{i} \bar{B}_{i} & \gamma I_{1} & 0 \\
0 & I & 0 & \gamma I_{1}
\end{array}\right]<0
$$

where

$$
\begin{gathered}
A_{11}=P \bar{A}_{i}+\bar{A}_{i}^{T} P-P K_{i p} \bar{C}_{i}-\left(P K_{i p} \bar{C}\right)^{T}+I_{e} \\
A_{12}=P \bar{D}_{i}+\left[Q\left(K_{i l} \bar{C}_{i}+K_{i v} \bar{C}_{i}\left(\bar{A}_{i}-K_{i p} \bar{C}_{i}\right)\right)\right]^{T}, \\
A_{22}=Q K_{i v} \bar{C}_{i} \bar{D}_{i}+\left(Q K_{i v} \bar{C}_{i} \bar{D}_{i}\right)^{T}+I_{s}
\end{gathered}
$$

hold, the system is stable and the $H_{\infty}$ performance is guaranteed with an attenuation level $\gamma$.

Theorem 5. Based on Lemma 4, given $\gamma>0$ and error system model (18), if there exist symmetric positive-definite matrices $P$ and $Q$ and matrices $K_{i p}, K_{i l}$, and $K_{i v}$ as well as matrix LMI such that (20) holds, then system (18) is robust asymptotically stable and satisfies the $H_{\infty}$ performance indicator as follows:

$$
\left\|e_{i s}\right\|^{2} \leq \gamma\left\|z_{i s}\right\|^{2}+V(0),
$$

where $\left\|e_{i s}\right\|^{2}=\int_{0}^{t_{1}}\left(e_{i s}{ }^{T} e_{i s}\right) d t,\left\|z_{i s}\right\|^{2}=\int_{0}^{t_{1}}\left(z_{i s}{ }^{T} z_{i s}\right) d t$, and $I_{e}, I_{s}$, and $I_{1}$ are unit matrices.

Proof. Choosing the Lyapunov candidate as $V=e_{i s}^{T} P_{1} e_{i s}$, combine (16) along with the time derivative of $V$ is given by

$$
\dot{V}=e_{i s}^{T}\left(A_{i s}^{T} P_{1}+P_{1} A_{i s}\right) e_{i s}+2 e_{i s}^{T} P_{1} N_{i s} z_{i s} .
$$

Consider the following index:

$$
J=\int_{0}^{t_{1}}\left(e_{i s}^{T} e_{i s}-\gamma z_{i s}^{T} z_{i s}\right) d t
$$

Thus,

$$
\begin{aligned}
J= & \int_{0}^{t_{1}}\left(e_{i s}^{T} e_{i s}-\gamma z_{i s}^{T} z_{i s}+\dot{V}\right) d t-\int_{0}^{t_{1}} \dot{V} d t \\
= & \int_{0}^{t_{1}}\left(e_{i s}^{T} e_{i s}-\gamma z_{i s}^{T} z_{i s}+\dot{V}\right) d t-V\left(t_{1}\right)+V(0) \\
\leq & \int_{0}^{t_{1}}\left(e_{i s}^{T} e_{i s}-\gamma z_{i s}^{T} z_{i s}+\dot{V}\right) d t+V(0) \\
= & \int_{0}^{t_{1}}\left[e_{i s}^{T}\left(A_{i s}^{T} P_{1}+P_{1} A_{i s}+I\right) e_{i s}\right. \\
& \left.+e_{i s}^{T} P_{1} N_{i} z_{i s}-\gamma z_{i s}^{T} z_{i s}\right] d t+V(0) \\
= & \int_{0}^{t_{1}}\left(\left[\begin{array}{ll}
e_{i s} \\
z_{i s}
\end{array}\right]^{T}\left[\begin{array}{cc}
A_{i s}^{T} P_{1}+P_{1} A_{i s}+I & P_{1} N_{i} \\
N_{i}^{T} P_{1} & -\gamma I_{1}
\end{array}\right]\left[\begin{array}{l}
e_{i s} \\
z_{i s}
\end{array}\right]\right) d t \\
& +V(0) .
\end{aligned}
$$

Considering that the estimation error is bounded, define the following inequality:

$$
\left[\begin{array}{cc}
A_{i s}^{T} P_{1}+P_{1} A_{i s}+I & P_{1} N_{i s} \\
N_{i s}^{T} P_{1} & -\gamma I_{1}
\end{array}\right]<0 .
$$

Next, define symmetric positive-definite matrix $P_{1}=$ $\left[\begin{array}{ll}P & 0 \\ 0 & I\end{array}\right]$; then,

$$
\begin{gathered}
P_{1} N_{i s}=\left[\begin{array}{cc}
P \bar{B}_{i} & 0 \\
K_{i v} \bar{C}_{i} \bar{B}_{i} & I
\end{array}\right], \\
A_{i s}^{T} P_{1}+P_{1} A_{i s}+I=\left[\begin{array}{cc}
A_{11} & A_{12} \\
A_{12}^{T} & A_{22}
\end{array}\right],
\end{gathered}
$$

where $A_{11}, A_{12}$, and $A_{22}$ are defined in (21); then,

$$
\int_{0}^{t_{1}}\left(e_{i s}^{T} e_{i s}\right) d t \leq \int_{0}^{t_{1}} \gamma z_{i s}^{T} z_{i s} d t+V(0)
$$

Inequality (22) can be obtained. Therefore, the observer satisfies the $H_{\infty}$ performance indicator and this completes the proof of Theorem 5 .

3.2. Actuator Fault Observer Design. This subsection designs the actuator fault estimator, along with the observer driven by the corrected (sensor fault compensated) output and control signals. Therefore, the system given in (3) can be converted to

$$
\bar{S}_{i f:}:\left\{\begin{aligned}
\dot{\bar{x}}_{i}= & \bar{A}_{i} \bar{x}_{i}+\bar{B}_{i} \\
& \cdot\left[f_{i}\left(q_{i}, \dot{q}_{i}\right)+g_{i}\left(q_{i}\right)\left(u_{i}+f_{i a}\right)+h_{i}(q, \dot{q}, \ddot{q})\right] \\
\bar{y}_{i}= & \bar{C}_{i} \bar{x}_{i}+\bar{D}_{i} e_{f i s} .
\end{aligned}\right.
$$

Equivalently, design another DPIO as (30) to simultaneously estimate the system states and actuator fault:

$$
\widehat{\bar{S}}_{i f}:\left\{\begin{array}{c}
\dot{\overline{\bar{x}}}_{i}=\bar{A}_{i} \hat{\bar{x}}_{i}+\bar{B}_{i}\left[\widehat{f}_{i}\left(\widehat{q}_{i}, \dot{\hat{q}}_{i}\right)+\widehat{g}_{i}\left(\widehat{q}_{i}\right)\left(u_{i}+\widehat{f}_{i a}\right)\right. \\
\left.\quad+v_{i}-\beta_{i} \operatorname{sgn}\left(e_{i}^{T} P_{i} \bar{B}_{i}\right)\right] \\
\quad+\bar{K}_{i p}\left(\bar{y}_{i}-\widehat{\bar{y}}_{i}+\bar{D}_{i} e_{f i s}\right), \\
\hat{\bar{y}}_{i}=\bar{C}_{i} \widehat{\bar{x}}_{i}+\bar{D}_{i} e_{f i s}, \\
\dot{\hat{f}}_{i a}=\bar{K}_{i l} \bar{C}_{i} \dot{e}_{i x}+\bar{K}_{i v} \bar{C}_{i} e_{i x} .
\end{array}\right.
$$


By using (29) and (30), the error dynamics can be changed as follows:

$$
\begin{aligned}
\dot{e}_{i}= & \dot{\bar{x}}_{i}-\dot{\overline{\bar{x}}}_{i} \\
= & \left(\bar{A}_{i}-\bar{K}_{i p} \bar{C}_{i}\right) e_{i}+\bar{K}_{i p} \bar{D}_{i} e_{f i s} \\
& +\bar{B}_{i} m_{a}+\bar{B}_{i} g_{i} e f_{i a}, \\
\dot{e}_{f i a}= & \dot{f}_{i a}-\dot{\hat{f}}_{i a} \\
= & \dot{f}_{i a}-\left[\bar{K}_{i l} \bar{C}_{i}+\bar{K}_{i v} \bar{C}_{i}\left(\bar{A}_{i}-\bar{K}_{i p} \bar{C}_{i}\right)\right] e_{i} \\
& +\bar{K}_{i v} \bar{C}_{i} \bar{K}_{i p} \bar{D}_{i} e_{f i s}+\bar{K}_{i v} \bar{C}_{i} \bar{B}_{i} m_{a} \\
& +\bar{K}_{i v} \bar{C}_{i} \bar{B}_{i} g_{i} e f_{i a},
\end{aligned}
$$

where

$$
\begin{gathered}
m_{a}=\left[\left(f_{i}-\widehat{f}_{i}\right)+\left(g_{i}-\widehat{g}_{i}\right)\left(u_{i}+\widehat{f}_{a}\right)\right. \\
\left.-v_{i}+h_{i}+\beta_{i} \operatorname{sgn}\left(e_{i}^{T} P_{i} \bar{B}_{i}\right)\right] .
\end{gathered}
$$

Therefore, the augmented estimator will then be of the following form:

$$
\dot{e}_{i a}=A_{i a} e_{i a}+N_{i a} z_{i a}+E_{i a} \bar{B}_{i} m_{a}
$$

where $e_{i a}=\left[\begin{array}{c}e_{i} \\ e_{f i a}\end{array}\right]$ and

$$
\begin{gathered}
A_{i a}=\left[\begin{array}{cc}
\bar{A}_{i}-\bar{K}_{i p} \bar{C}_{i} & \bar{B}_{i} g_{i} \\
-\bar{K}_{i l} \bar{C}_{i}-\bar{K}_{i v} \bar{C}_{i}\left(\bar{A}_{i}-K_{p a} \bar{C}_{i}\right) & \bar{K}_{i v} \bar{C}_{i} \bar{B}_{i} g_{i}
\end{array}\right], \\
N_{i a}=\left[\begin{array}{cc}
\bar{K}_{i p} \bar{D}_{i} & 0 \\
\bar{K}_{i v} \bar{C}_{i} \bar{K}_{i p} \bar{D}_{i} & I
\end{array}\right], \\
z_{i a}=\left[\begin{array}{c}
e_{f i s} \\
\dot{f}_{i a}
\end{array}\right], \quad E_{i a}=\left[\begin{array}{c}
I \\
\bar{K}_{i v} \bar{C}_{i}
\end{array}\right] .
\end{gathered}
$$

Theorem 6. Also based on Lemma 4, given $\gamma>0$ and error system model (31), if there exist symmetric positive-definite matrices $P$ and $Q$ and matrices $K_{P a}, K_{i a}$, and $K_{v a}$ as well as matrix LMI such that

$$
\left[\begin{array}{ccccc}
A_{11} & A_{12} & P \bar{K}_{i p} \bar{D}_{i} & 0 & P \\
* & A_{22} & \bar{K}_{i v} \bar{C}_{i} \bar{K}_{i p} \bar{D}_{i} & I & \bar{K}_{i v} \bar{C}_{i} \\
* & * & \gamma I_{1} & 0 & 0 \\
* & * & * & \gamma I_{1} & 0 \\
* & * & * & * & \gamma I_{1}
\end{array}\right]<0,
$$

where

$$
\begin{gathered}
A_{11}=P \bar{A}_{i}+\bar{A}_{i}^{T} P-P \bar{K}_{i p} \bar{C}_{i}-\left(P \bar{K}_{i p} \bar{C}\right)^{T}+I_{e}, \\
A_{12}=P \bar{B}_{i} g_{i}+\left[Q\left(-\bar{K}_{i l} \bar{C}_{i}-\bar{K}_{i v} \bar{C}_{i}\left(\bar{A}_{i}-\bar{K}_{i p} \bar{C}_{i}\right)\right)\right]^{T}, \\
A_{22}=Q \bar{K}_{i v} \bar{C}_{i} \bar{B}_{i} g_{i}+\left(Q \bar{K}_{i v} \bar{C}_{i} \bar{B}_{i} g_{i}\right)^{T}+I_{s}
\end{gathered}
$$

hold, then system (31) is robust asymptotically stable and satisfies the $H_{\infty}$ performance indicator as follows:

$$
\left\|e_{i a}\right\|^{2} \leq \gamma\left\|z_{i a}\right\|^{2}+V(0),
$$

where $\left\|e_{i a}\right\|^{2}=\int_{0}^{t_{1}}\left(e_{i a}{ }^{T} e_{i a}\right) d t,\left\|z_{i a}\right\|^{2}=\int_{0}^{t_{1}}\left(z_{i a}{ }^{T} z_{i a}\right) d t$, and $I_{e}$, $I_{s}$, and $I_{1}$ are unit matrices.

Proof. The proof procedure of Theorem 6 is similar to that of Theorem 5; here it is omitted.

\section{Active Decentralized Fault-Tolerant Controller Design}

In this section, the ADFTC based on dynamic output feedback is designed to ensure the stability and tracking accuracy of a reconfigurable manipulator with acting actuator and sensor faults concurrently.

Considering the faulty subsystem dynamic model (29), the decentralized fault-tolerant controller is designed as follows:

$$
\begin{gathered}
\dot{x}_{i c}=A_{i c} x_{i c}+B_{i c} \bar{y}_{i}, \\
u_{i}=C_{i c} x_{i c}+D_{i c} \bar{y}_{i}-\widehat{f}_{i a} .
\end{gathered}
$$

Aggregation of (29) and (38) gives the following system:

$$
\begin{gathered}
\dot{x}_{i l}=A_{i l} x_{i l}+E_{i l} d_{i l}+W_{i l} \bar{B}_{i}, \\
\bar{y}_{i}=C_{i l} x_{i l}+D_{i l} d_{i l},
\end{gathered}
$$

where

$$
\begin{gathered}
x_{i l}=\left[\begin{array}{c}
\bar{x}_{i} \\
x_{i c}
\end{array}\right], \quad A_{i l}=\left[\begin{array}{cc}
\bar{A}_{i}+\bar{B}_{i} g_{i} D_{i c} \bar{C}_{i} & \bar{B}_{i} g_{i} C_{i c} \\
B_{i c} \bar{C}_{i} & A_{i c}
\end{array}\right], \\
d_{i l}=\left[\begin{array}{c}
e_{f i a} \\
e_{f i s}
\end{array}\right], \quad W_{i l}=\left[\begin{array}{c}
f_{i}+h_{i} \\
0
\end{array}\right], \\
E_{i l}=\left[\begin{array}{cc}
\bar{B}_{i} g_{i} & \bar{B}_{i} g_{i} D_{i c} \bar{D}_{i} \\
0 & B_{i c} \bar{D}_{i}
\end{array}\right], \\
C_{i l}=\left[\begin{array}{ll}
\bar{C}_{i} & 0
\end{array}\right], \quad D_{i l}=\left[\begin{array}{ll}
0 & \bar{D}_{i}
\end{array}\right] .
\end{gathered}
$$

Similarly, based on Lemma 4, the existing condition of dynamic output feedback robust control is given in Theorem 7.

Theorem 7. Given $\gamma>0$ and fault subsystems dynamic model (29), if there exist symmetric positive-definite matrices $P$ and $Q$ and matrices $A_{i c}, B_{i c}, C_{i c}$, and $D_{i c}$ as well as matrix LMI such that

$$
\left[\begin{array}{ccccc}
A_{11} & A_{12} & P \bar{B}_{i} g_{i} & P\left(\bar{B}_{i} g_{i} D_{i c} \bar{D}_{i}\right) & P\left(f_{i}+h_{i}\right) \\
A_{12}^{T} & A_{22} & 0 & B_{i c} \bar{D}_{i} & 0 \\
* & * & \gamma I & 0 & 0 \\
* & * & * & \gamma I & 0 \\
* & * & * & * & \gamma I
\end{array}\right]<0,
$$


where

$$
\begin{gathered}
A_{11}=P \bar{A}_{i}+\bar{A}_{i}^{T} P+P \bar{B}_{i} g_{i} D_{i c} \bar{C}_{i} \\
+\left(P \bar{B}_{i} g_{i} D_{i c} \bar{C}_{i}\right)^{T}+I_{e}, \\
A_{12}=P \bar{B}_{i} g_{i} C_{i c}+\left(Q B_{i c} \bar{C}_{i}\right)^{T}, \\
A_{22}=Q A_{i c}+\left(Q A_{i c}\right)^{T}+I_{s}
\end{gathered}
$$

hold, then the closed-loop system (39) is robust asymptotically stable and satisfies the $H_{\infty}$ performance indicator as follows:

$$
\left\|x_{i l}\right\|^{2} \leq \gamma\left\|d_{i}\right\|^{2}+V(0),
$$

where $\left\|x_{i l}\right\|^{2}=\int_{0}^{t_{1}}\left(x_{i l}^{T} x_{i l}\right) d t,\left\|d_{i}\right\|^{2}=\int_{0}^{t_{1}}\left(d_{i}^{T} d_{i}\right) d t$, and $I_{e}, I_{s}$, and $I_{1}$ are unit matrices.

Proof. Choosing the candidate function $V=x_{i l}^{T} P_{1} x_{i l}$, along with the trajectories of (39) is then given by

$$
\begin{aligned}
\dot{V}= & x_{i l}^{T}\left(A_{i l}^{T} P_{1}+P_{1} A_{i l}\right) x_{i l}+x_{i l}^{T} P_{1} E_{i l} d_{i} \\
& +d_{i}^{T} E_{i l}^{T} P_{1} x_{i l}+2 x_{i l}^{T} P_{1} W_{i l} \bar{B}_{i} .
\end{aligned}
$$

Given the index

$$
J=\int_{0}^{t_{1}}\left(x_{i l}^{T} x_{i l}-\gamma d_{i l}^{T} d_{i l}\right) d t,
$$

thus,

$$
\begin{aligned}
& J=\int_{0}^{t_{1}}\left(x_{i l}^{T} x_{i l}-\gamma d_{i}^{T} d_{i l}+\dot{V}\right) d t-\int_{0}^{t_{1}} \dot{V} d t \\
& =\int_{0}^{t_{1}}\left(x_{i l}^{T} x_{i l}-\gamma d_{i}^{T} d_{i l}+\dot{V}\right) d t-V\left(t_{1}\right)+V(0) \\
& \leq \int_{0}^{t_{1}}\left(x_{i l}^{T} x_{i l}-\gamma d_{i}^{T} d_{i l}+\dot{V}\right) d t+V(0) \\
& =\int_{0}^{t_{1}}\left[x_{i l}^{T}\left(A_{i l}^{T} P_{1}+P_{1} A_{i l}+I\right) x_{i l}\right. \\
& +x_{i l}^{T} P_{1} E_{i l} d_{i}+d_{i}^{T} E_{i l}^{T} P_{1} x_{i l} \\
& \left.+2 x_{i l}^{T} P_{1} W_{i l} \bar{B}_{i}-\gamma d_{i}^{T} d_{i}\right] d t+V(0) \\
& =\int_{0}^{t_{1}}\left(\left[\begin{array}{c}
x_{i l} \\
d_{i} \\
\bar{B}_{i}
\end{array}\right]^{T}\left[\begin{array}{ccc}
A_{i l}^{T} P_{1}+P_{1} A_{i l}+I & P_{1} E_{i l} & P_{1} W_{i l} \\
P_{1} E_{i l}^{T} & -\gamma I_{1} & 0 \\
P_{1} W_{i l}^{T} & 0 & -\gamma I_{1}
\end{array}\right]\right. \\
& \left.\cdot\left[\begin{array}{c}
x_{i l} \\
d_{i} \\
\bar{B}_{i}
\end{array}\right]\right) d t+V(0) \text {. }
\end{aligned}
$$

Considering that estimation error is bounded, define the following inequality:

$$
\left[\begin{array}{ccc}
A_{i l}^{T} P_{1}+P_{1} A_{i l}+I & P_{1} E_{i l} & P_{1} W_{i l} \\
P_{1} E_{i l}^{T} & -\gamma I_{1} & 0 \\
P_{1} W_{i l}^{T} & 0 & -\gamma I_{1}
\end{array}\right]<0 .
$$

Next, define symmetric positive-definite matrix $P_{1}=$ $\left[\begin{array}{cc}P & 0 \\ 0 & I\end{array}\right]$; then,

$$
\begin{gathered}
P_{1} E_{i l}=\left[\begin{array}{cc}
P \bar{B}_{i} g_{i} & P \bar{B}_{i} g_{i} D_{i c} \bar{D}_{i} \\
0 & B_{i c} \bar{D}_{i}
\end{array}\right], \\
P_{1} W_{i l}=\left[\begin{array}{c}
P\left(f_{i}+h_{i}\right) \\
0
\end{array}\right], \\
A_{i s}^{T} P_{1}+P_{1} A_{i s}+I=\left[\begin{array}{cc}
A_{11} & A_{12} \\
A_{12}^{T} & A_{22}
\end{array}\right],
\end{gathered}
$$

where $A_{11}, A_{12}$, and $A_{22}$ are defined in (42); then,

$$
\int_{0}^{t_{1}}\left(x_{i l}^{T} x_{i l}\right) d t \leq \int_{0}^{t_{1}} \gamma d_{i}^{T} d_{i} d t+V(0)
$$

Inequality (43) can be obtained. Therefore, the system satisfies the $H_{\infty}$ performance indicator and this completes the proof of Theorem 7 .

\section{Simulation Results}

To verify the effectiveness of the proposed ADFTC strategy, in this subsection, two 3-DOF reconfigurable manipulators with different configurations shown in Figure 1 are employed to illustrate the simulation results.

The initial position and velocity are set as $q_{1}(0)=q_{2}(0)=$ $q_{3}(0)=1$ and $\dot{q}_{1}(0)=\dot{q}_{2}(0)=\dot{q}_{3}(0)=0$, respectively.

By solving the LMI conditions given in 1,2 , and 3 , the dynamic output feedback controller and observer gains are

$$
\begin{aligned}
& A_{1 c}=\left[\begin{array}{cccc}
1.7261 & 2.9781 & 4.4521 & 6.3562 \\
1.1781 & 2.1535 & 3.3562 & 5.3070 \\
-1.0682 & -1.5344 & 4.5082 & 5.1247 \\
-0.9344 & -1.4606 & 3.1247 & 4.1075
\end{array}\right], \\
& A_{2 c}=\left[\begin{array}{cccc}
1.0261 & 2.8531 & 4.0951 & 5.9062 \\
1.1141 & 2.0031 & 3.1639 & 5.0250 \\
-1.0009 & -1.3614 & 4.1163 & 4.9428 \\
-0.8314 & -1.2171 & 2.7319 & 4.0014
\end{array}\right],
\end{aligned}
$$




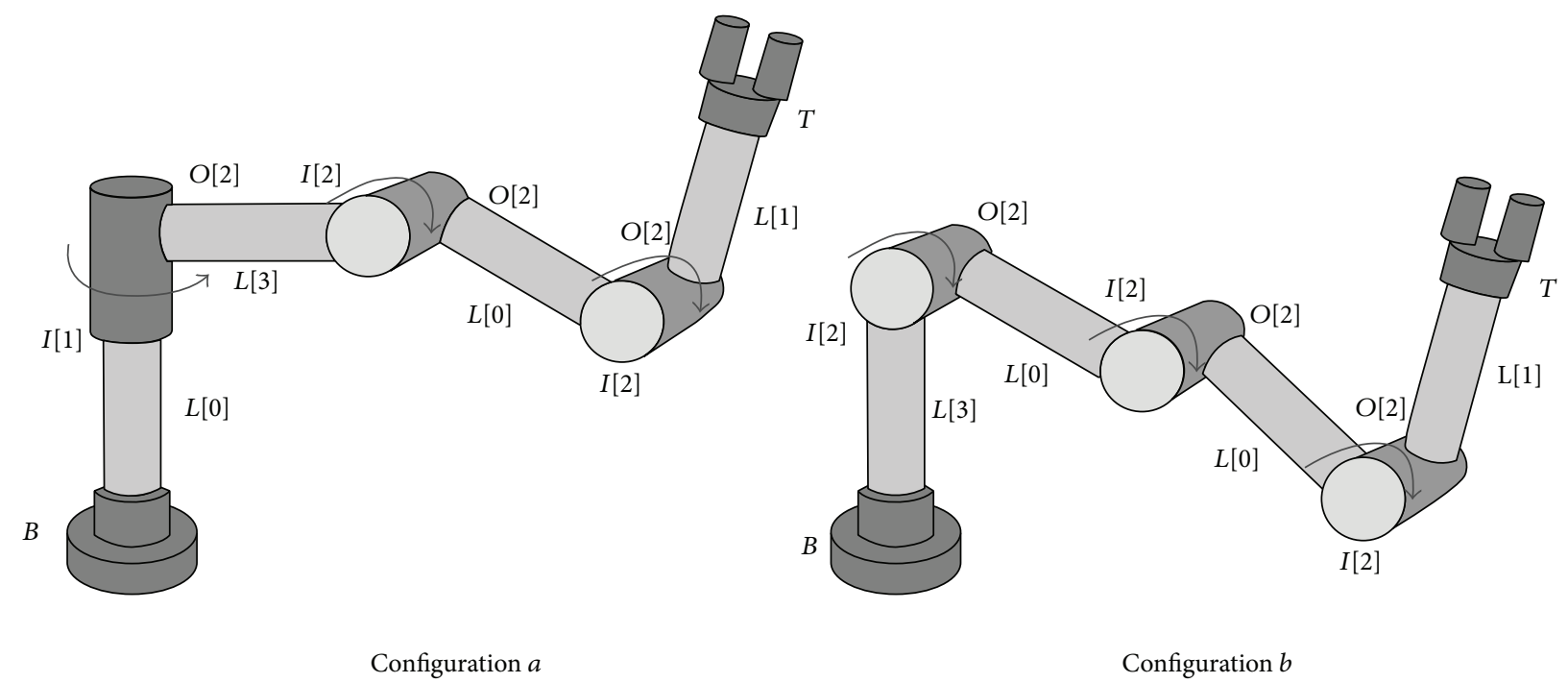

FIGURE 1: Configurations of 3-DOF reconfigurable manipulators.

$$
\begin{aligned}
& A_{3 c}=\left[\begin{array}{cccc}
0.9841 & 2.0781 & 4.0194 & 5.8376 \\
1.0781 & 1.9535 & 3.0062 & 4.9030 \\
-0.9718 & -1.0981 & 3.5462 & 5.0007 \\
-0.9004 & -0.9261 & 2.9654 & 9.9612
\end{array}\right], \\
& B_{1 c}=\left[\begin{array}{cccc}
21.5639 & 40.4866 & 40.4866 & 20.8926 \\
2.9584 & -22.8672 & 27.0025 & 44.1431 \\
24.9533 & 5.7852 & -8.2747 & 19.3143 \\
-5.9514 & 13.2063 & 38.8838 & 34.8115
\end{array}\right] \text {, } \\
& B_{2 c}=\left[\begin{array}{cccc}
21.1543 & 40.0039 & 39.8753 & 20.0034 \\
2.7635 & -22.0753 & 26.5025 & 43.9471 \\
24.6494 & 5.0735 & -8.0415 & 19.0001 \\
-5.6401 & 12.9083 & 38.1307 & 34.0671
\end{array}\right] \text {, } \\
& B_{3 c}=\left[\begin{array}{cccc}
20.9680 & 40.1496 & 39.4986 & 20.5639 \\
2.6835 & -22.0681 & 26.4517 & 43.1931 \\
24.2583 & 5.1327 & -8.0017 & 18.9153 \\
-5.3454 & 12.8963 & 38.0818 & 33.9911
\end{array}\right] \text {, } \\
& C_{1 c}=\left[\begin{array}{llll}
0.7261 & -1.0665 & -0.9665 & 0.3214
\end{array}\right], \\
& C_{2 c}=\left[\begin{array}{llll}
0.3274 & -0.7765 & -0.5665 & 0.2341
\end{array}\right], \\
& C_{3 c}=\left[\begin{array}{llll}
0.0661 & -0.1735 & -0.0913 & 0.0648
\end{array}\right], \\
& D_{1 c}=\left[\begin{array}{llll}
2.6557 & -1.9799 & -2.0486 & 5.1928
\end{array}\right] \text {, } \\
& D_{2 c}=\left[\begin{array}{llll}
1.9471 & -1.0006 & -2.0936 & 4.9718
\end{array}\right] \text {, } \\
& D_{3 c}=\left[\begin{array}{llll}
0.9557 & -0.8799 & -1.0486 & 3.1928
\end{array}\right] \text {. }
\end{aligned}
$$

The sensor fault observer gains are calculated as

$$
\begin{gathered}
K_{1 p}=\left[\begin{array}{cccc}
-14.2064 & 9.9445 & 8.5239 & 11.3651 \\
0.5600 & -12.4741 & 10.6921 & 14.2561 \\
7.4114 & 5.1880 & 4.4468 & -5.9291 \\
3.6582 & 2.5608 & 2.1949 & -2.9266
\end{array}\right], \\
K_{2 p}=\left[\begin{array}{cccc}
-12.2064 & 8.6255 & 7.9152 & 10.8921 \\
0.4725 & -11.0031 & 9.8513 & 12.9151 \\
6.4164 & 5.1880 & 4.3518 & -4.831 \\
2.6532 & 1.9375 & 2.1949 & -2.0216
\end{array}\right], \\
K_{3 p}=\left[\begin{array}{ccccc}
-12.2257 & 8.6415 & 7.9514 & 10.3621 \\
0.4651 & -12.4741 & 10.6921 & 14.2561 \\
6.9416 & 4.9480 & 3.9723 & -5.3611 \\
2.2418 & 1.5308 & 2.0009 & -2.5136
\end{array}\right], \\
K_{1 l}=\left[\begin{array}{lllll}
7.5289 & 3.1970 & 8.2071 & 5.5190
\end{array}\right], \\
K_{2 v}=\left[\begin{array}{lllll}
10.5289 & 5.1970 & 13.2071 & 6.5190
\end{array}\right], \\
K_{3 l}=\left[\begin{array}{lllll}
6.953258 & 4.0940 & 12.8070 & 5.9510
\end{array}\right],
\end{gathered}
$$



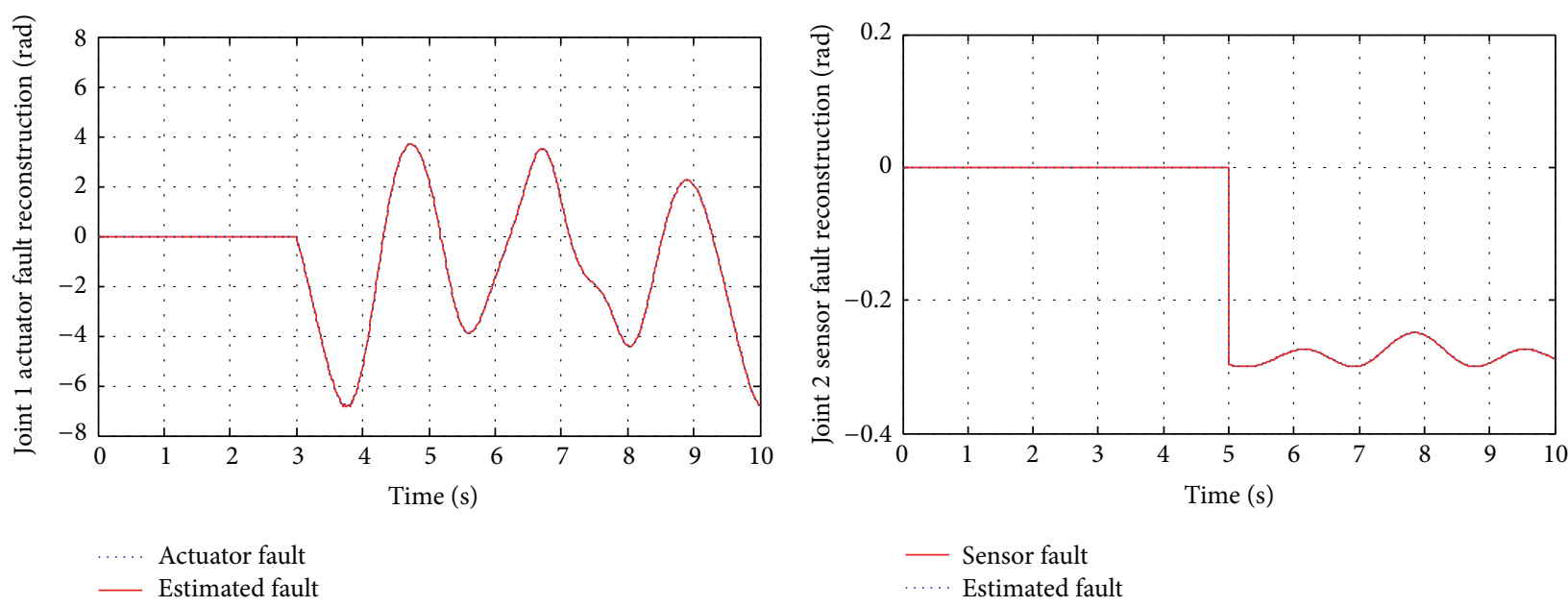

(a)
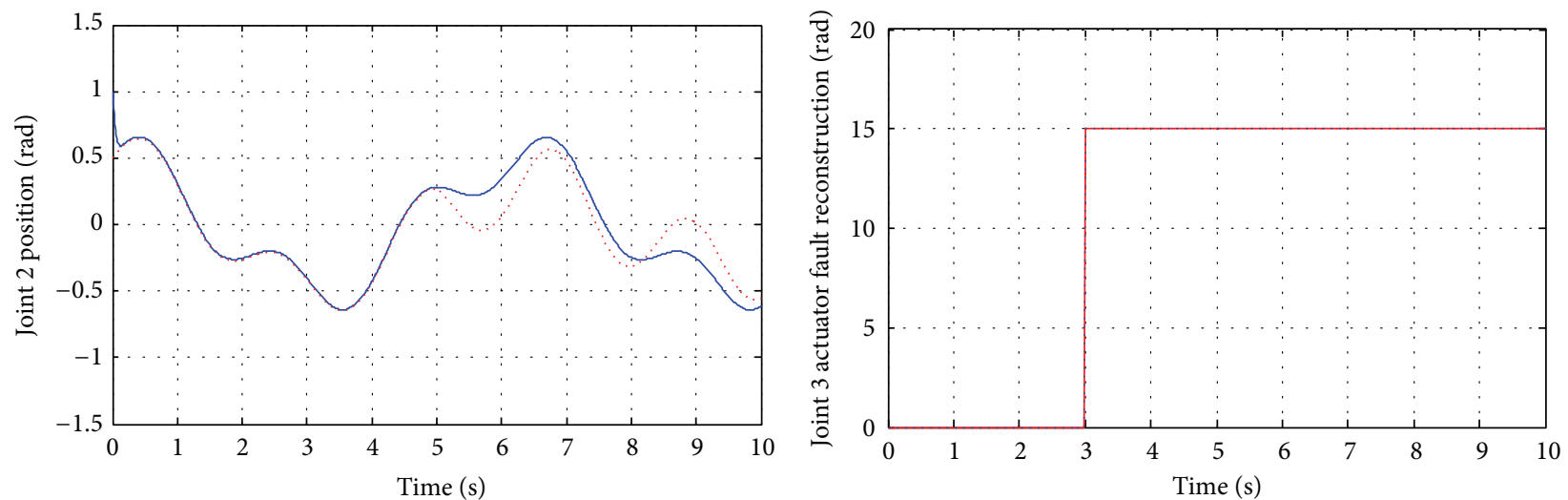

— Desired trajectory

Without sensor faults compensation trajectory

(c)

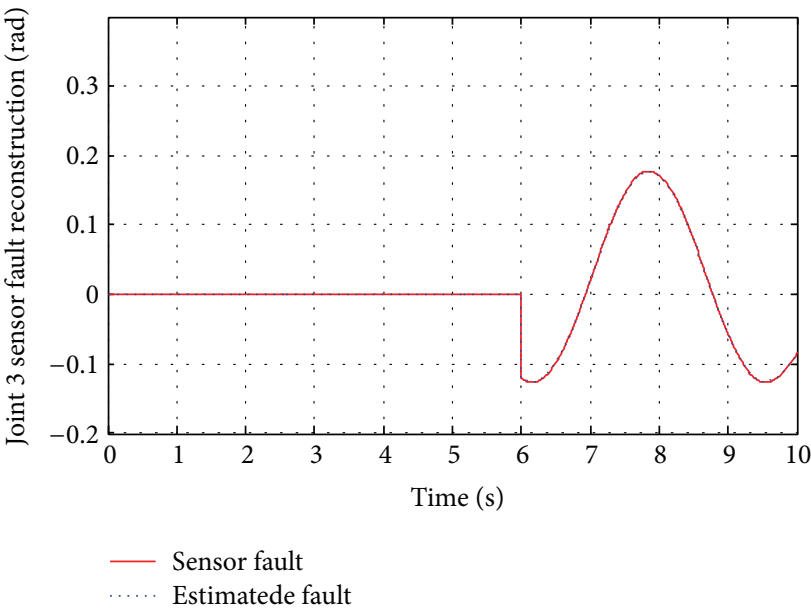

(e)

- Actuator fault Estimatede fault

(d)

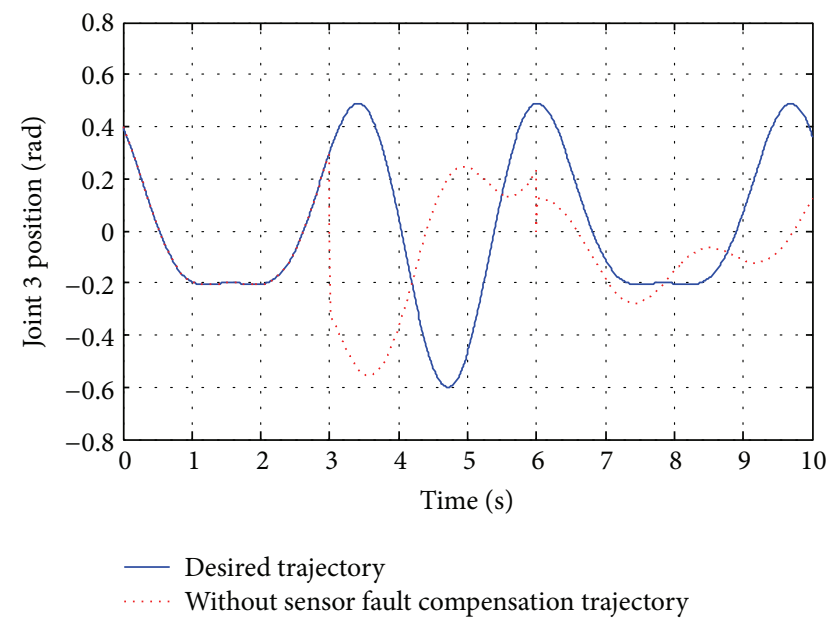

(f)

FIGURE 2: (a) Actuator fault estimation of joint 1; (b) sensor fault estimation of joint 2; (c) tracking performance with uncompensated sensor fault of joint 2; (d) actuator fault estimation of joint 3; (e) sensor fault estimation of joint 3; (f) tracking performance with uncompensated sensor fault of joint 3 . 


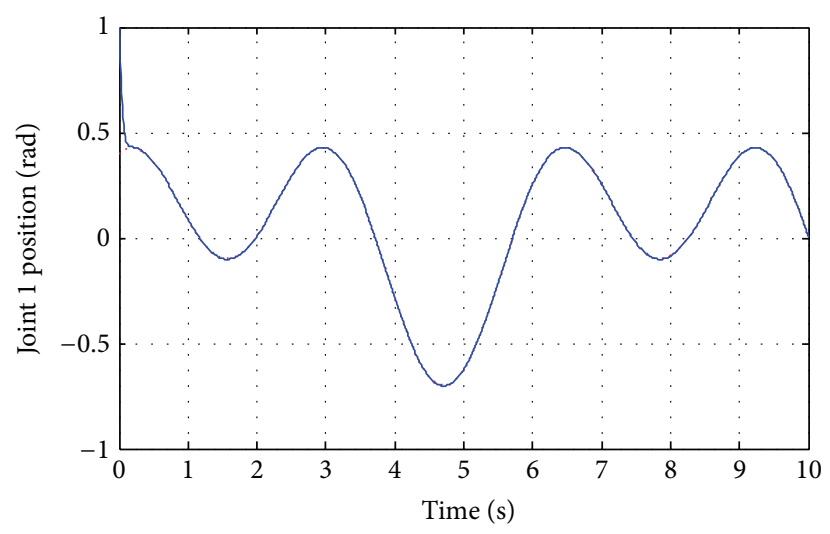

Desired trajectory — Actual trajectory

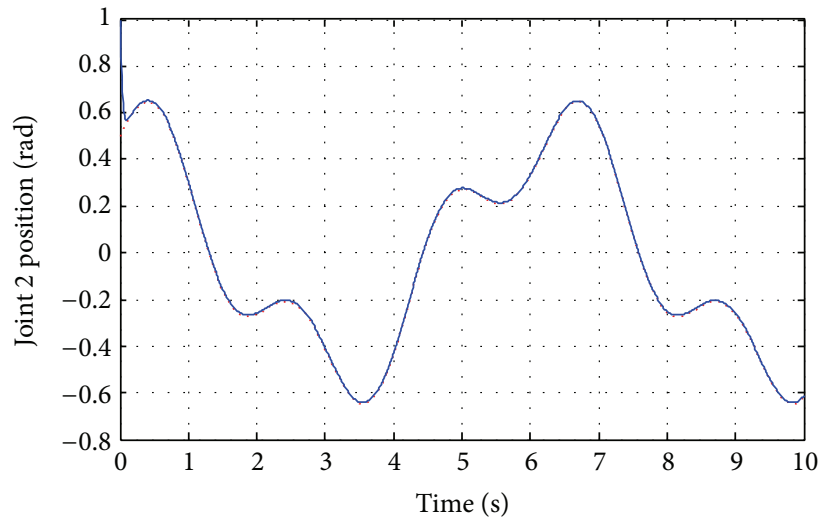

Desired trajectory

— Actual trajectory

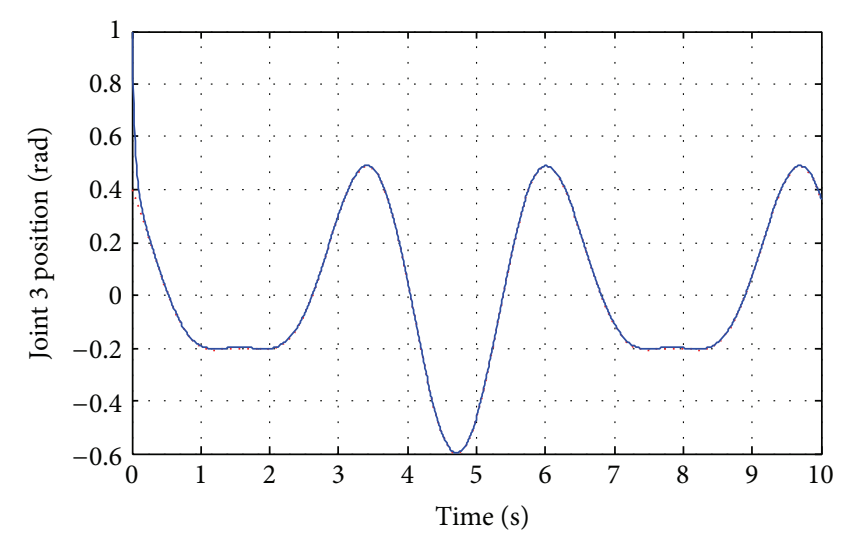

Desired trajectory

- Actual trajectory

FIgURE 3: The tolerant tracking performance of configuration $a$.

The actuator fault observer gains are calculated as

$$
\begin{aligned}
& \bar{K}_{1 p}=\left[\begin{array}{cccc}
27.1343 & -22.4461 & 15.7691 & 0.8612 \\
4.0364 & 0.1558 & 17.7803 & 1.1852 \\
14.1557 & 11.7100 & 8.2266 & -12.9699 \\
6.9872 & 5.7800 & 4.0606 & -6.4019
\end{array}\right], \\
& \bar{K}_{2 p}=\left[\begin{array}{cccc}
25.8643 & -20.4813 & 12.5191 & 0.7132 \\
4.0374 & 0.1098 & 13.7013 & 1.0352 \\
13.977 & 11.7100 & 8.0136 & -11.9819 \\
5.8924 & 5.1320 & 3.9836 & -5.3419
\end{array}\right], \\
& \bar{K}_{3 p}=\left[\begin{array}{cccc}
22.1463 & -20.4001 & 11.7251 & 0.702112 \\
4.0364 & 0.9958 & 12.9803 & 0.9562 \\
13.8937 & 11.7100 & 8.2266 & -12.9699 \\
5.8924 & 5.0972 & 3.9001 & -5.2919
\end{array}\right],
\end{aligned}
$$

$$
\begin{aligned}
& \bar{K}_{1 v}=\left[\begin{array}{llll}
6.5289 & 3.1970 & 5.2071 & 6.5190
\end{array}\right], \\
& \bar{K}_{2 v}=\left[\begin{array}{llll}
6.0963 & 3.0023 & 5.1325 & 6.2351
\end{array}\right], \\
& \bar{K}_{3 v}=\left[\begin{array}{llll}
5.9235 & 2.8971 & 5.0701 & 6.1180
\end{array}\right], \\
& \bar{K}_{1 l}=\left[\begin{array}{llll}
5.0231 & 3.5576 & 7.5657 & 6.4152
\end{array}\right], \\
& \bar{K}_{2 l}=\left[\begin{array}{llll}
4.9421 & 3.0893 & 7.1097 & 6.2321
\end{array}\right], \\
& \bar{K}_{3 l}=\left[\begin{array}{llll}
4.8511 & 2.9416 & 6.5725 & 6.0757
\end{array}\right] .
\end{aligned}
$$

Here, the control law (39) is applied to the whole control system, and the control parameters are selected as $\eta_{i f}=0.002$, $\eta_{i g}=0.002$, and $\eta_{i p}=500$, and the $H_{\infty}$ performance indicator is defined as $\gamma=1.0$.

First, considering configuration $a$, fault signals are added to the actuators of joint 1 , the position sensor of joint 2 , and both velocity sensor and actuator of joint 3 at $t=3 \mathrm{~s}, t=5 \mathrm{~s}$, $t=6 \mathrm{~s}$, and $t=3 \mathrm{~s}$, respectively. 


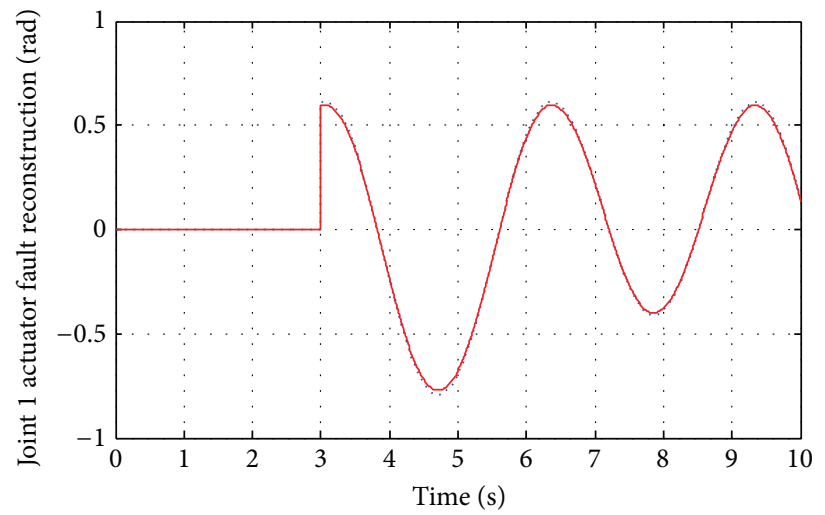

… Actuator fault

— Estimatede fault

(a)

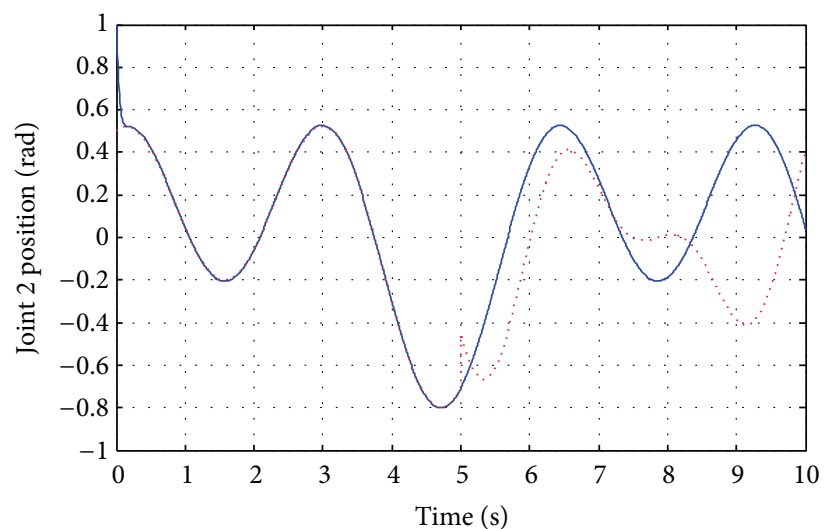

Desired trajectory

Without sensor fault compensatsion trajectory

(c)

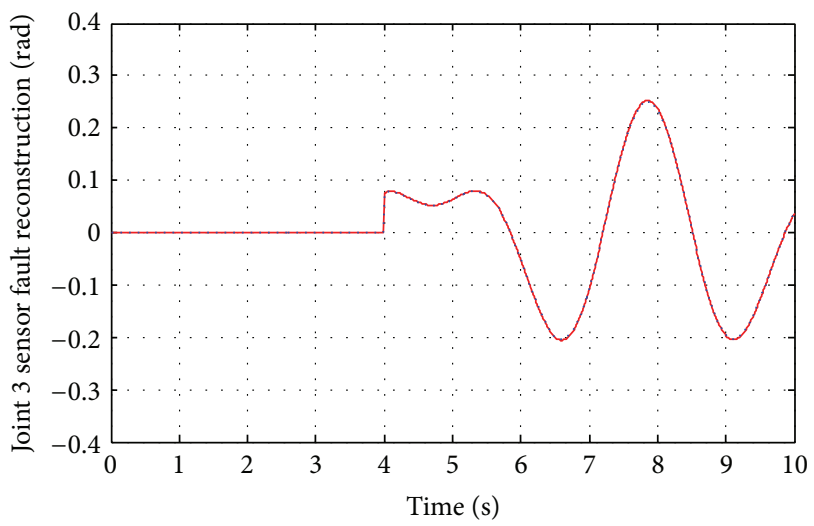

Sensor fault

Estimatede fault

(e)

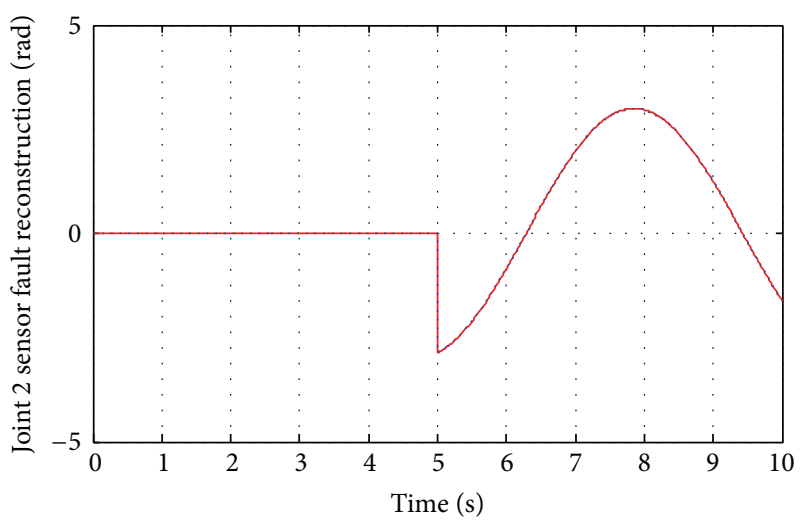

— Sensor fault

Estimatede fault

(b)

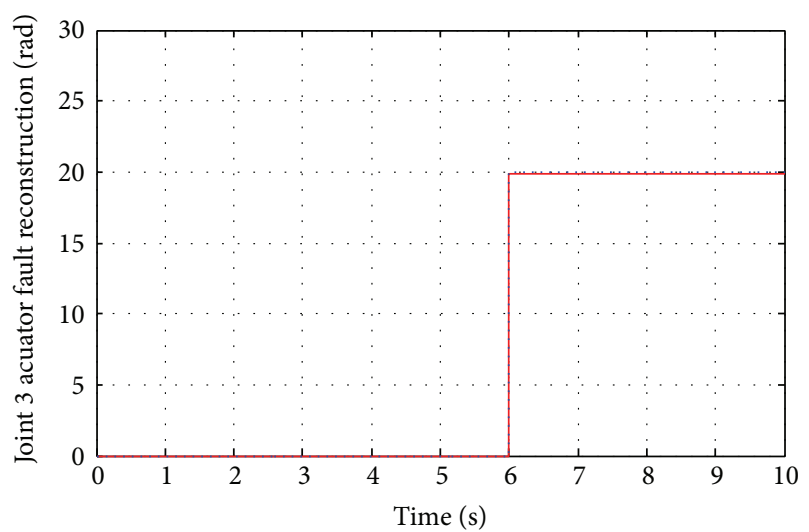

— Actuator fault

Estimated fault

(d)

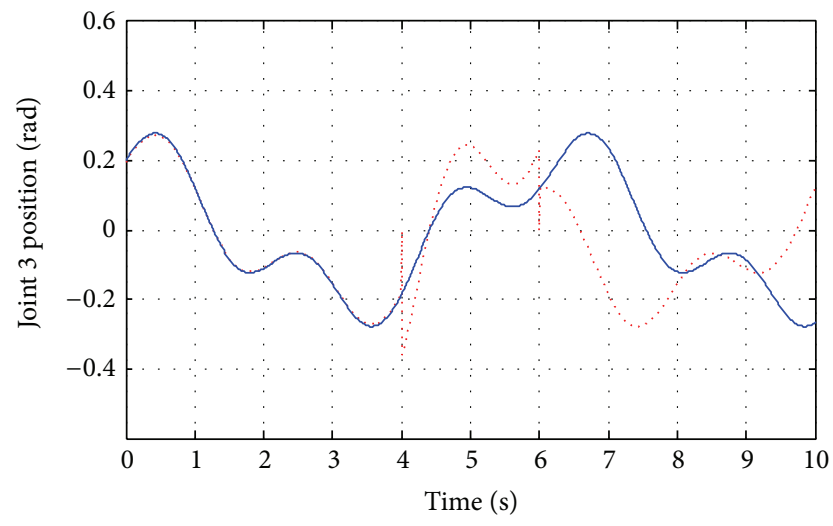

..... Without sensor fault compensation trajectory

— Desired trajectory

(f)

Figure 4: (a) Actuator fault estimation of joint 1; (b) sensor fault estimation of joint 2; (c) tracking performance with uncompensated sensor fault of joint 2; (d) actuator fault estimation of joint 3; (e) sensor fault estimation of joint 3; (f) tracking performance with uncompensated sensor fault of joint 3 . 


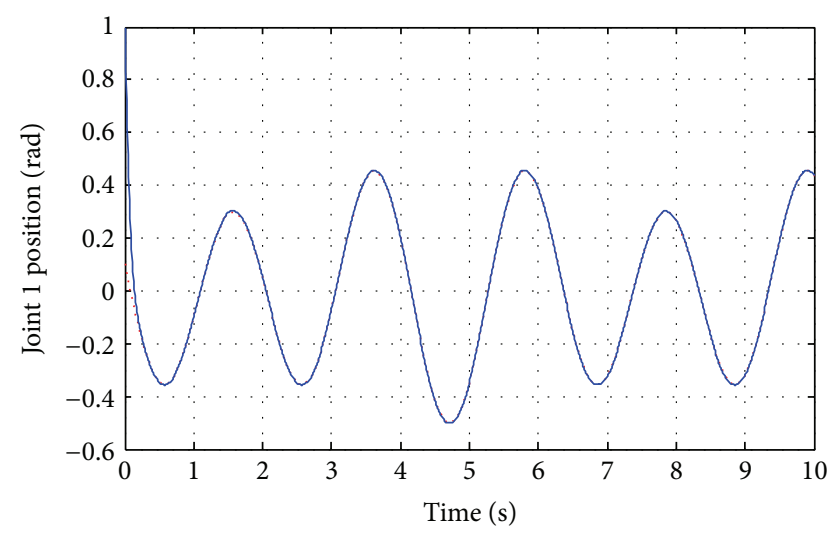

Desired trajectory - Actual trajectory

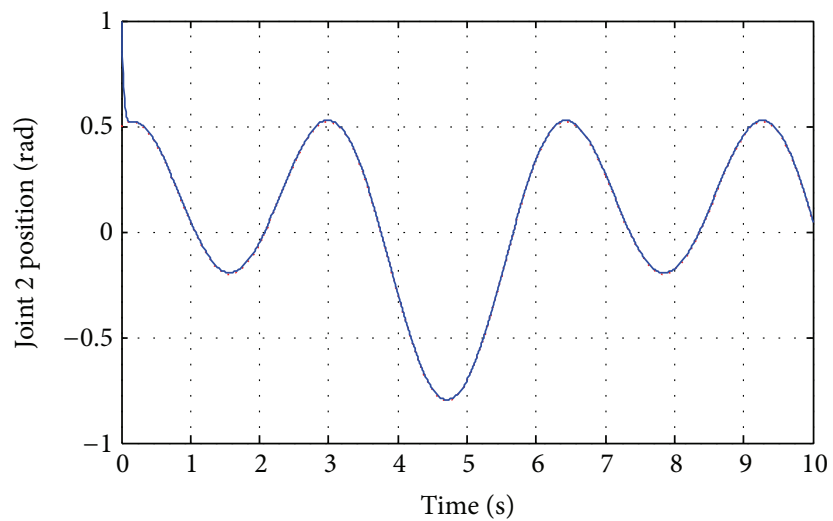

Desired trajectory

- Actual trajectory

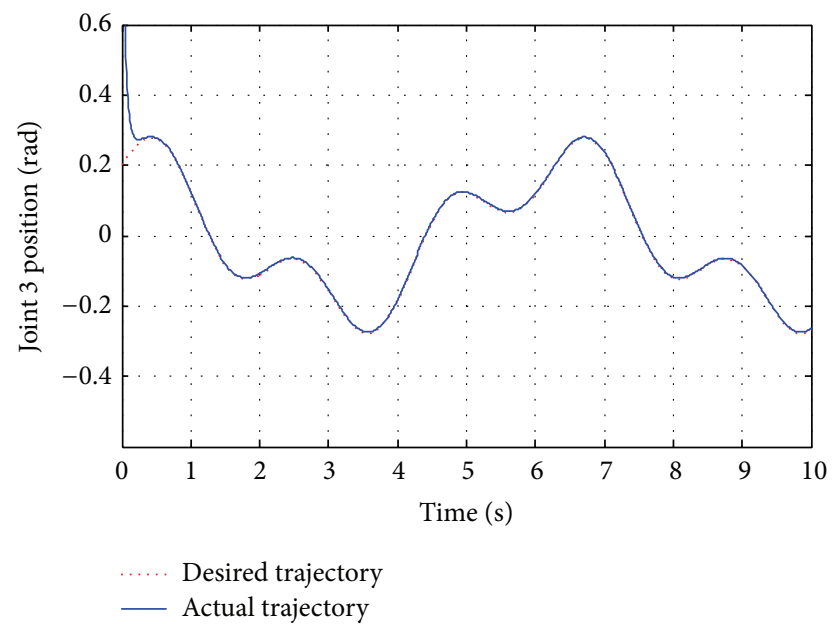

FIGURE 5: The tolerant tracking performance of configuration $b$.

The fault functions are as follows:

$$
\begin{gathered}
f_{1 a}= \begin{cases}0, & t \leq 3, \\
5 \sin \left(q_{1}\right) \dot{q}_{1}, & t>3,\end{cases} \\
f_{2 s}= \begin{cases}0, & t \leq 5, \\
-0.3 \cos \left(q_{2}\right), & t>5,\end{cases} \\
f_{3 a}= \begin{cases}0, & t \leq 3, \\
15, & t>3,\end{cases} \\
f_{3 s}= \begin{cases}0, & t \leq 6, \\
-0.3 q_{2}, & t>6 .\end{cases}
\end{gathered}
$$

As illustrated in Figure 2, from Figures 2(a), 2(b), 2(d), and $2(\mathrm{e})$ it can be obtained that the sensor faults and actuator faults can be detected online in real time when the faults occur via DPIO, and the actual trajectory cannot track the desired trajectory due to without sensor fault compensation from Figures 2(c) and 2(f). Figure 3 shows the tolerant tracking performance of configuration $a$, in which it can be seen that the actual trajectories can follow the desired trajectories in each joint.

To further test the effectiveness of the proposed scheme under different configurations, the same scheme applies to configuration $b$.

The fault functions are as follows:

$$
\begin{gathered}
f_{1 a}= \begin{cases}0, & t \leq 3, \\
2 \sin \left(q_{1}\right), & t>3,\end{cases} \\
f_{2 s}= \begin{cases}0, & t \leq 5, \\
3 \sin (t), & t>5,\end{cases} \\
f_{3 a}= \begin{cases}0, & t \leq 6, \\
20, & t>6,\end{cases} \\
f_{3 s}= \begin{cases}0, & t \leq 4, \\
-0.5 q_{2}, & t>4 .\end{cases}
\end{gathered}
$$


The simulation results are shown as in Figures 4 and 5 that illustrate that the proposed active decentralized fault-tolerant control can be applied to different configurations of reconfigurable manipulator without any parameters modification.

\section{Conclusion}

The paper develops a novel tolerant control strategy for reconfigurable manipulator with simultaneous actuator and sensor faults based on dynamic output feedback control. Using LMI technology, the DPIO is designed to estimate and compensate the actuator and sensor faults on line, and the estimator of actuator fault is put into the designed dynamic output feedback controller to realize active fault-tolerant control. The method obviates the disturbance of time delay for system by cancelling the need for the use of a fault diagnosis and isolation (FDI) unit and the controller not only can make fault system robust stability, but also can meet the requirement of $H_{\infty}$ performance indicators. The effectiveness of the proposed scheme is verified under the conditions of different configurations without modifying any parameter.

\section{Conflict of Interests}

The authors declare that there is no conflict of interests regarding the publication of this paper.

\section{Acknowledgment}

This work is supported by the National Natural Science Foundation of China under Grants 61374051 and 60974010 and the Scientific and Technological Development Plan Project in Jilin Province of China under Grant 20150520112JH.

\section{References}

[1] D. Rupp, G. Ducard, E. Shafai, and H. P. Geering, "Extended multiple model adaptive estimation for the detection of sensor and actuator faults," in Proceedings of the 44th IEEE Conference on Decision and Control, and the European Control Conference (CDC-ECC '05), pp. 3079-3084, December 2005.

[2] M. Du, J. Nease, and P. Mhaskar, "An integrated fault diagnosis and safe-parking framework for fault-tolerant control of nonlinear systems," International Journal of Robust and Nonlinear Control, vol. 22, no. 1, pp. 105-122, 2012.

[3] Y. Jiang, Q. Hu, and G. Ma, "Adaptive backstepping faulttolerant control for flexible spacecraft with unknown bounded disturbances and actuator failures," ISA Transactions, vol. 49, no. 1, pp. 57-69, 2010.

[4] D. Brambilla, L. M. Capisani, A. Ferrara, and P. Pisu, "Second order sliding mode observers for fault detection of robot manipulators," in Proceedings of the 47th IEEE Conference on Decision and Control (CDC '08), pp. 2949-2954, Cancún, Mexico, December 2008.

[5] S. T. Huang, E. J. Davison, and R. . Kwong, "Decentralized robust servomechanism problem for large flexible space structures under sensor and actuator failures," IEEE Transactions on Automatic Control, vol. 57, no. 12, pp. 3219-3224, 2012.
[6] J. Jiang and Y. Zhang, "Accepting performance degradation in fault-tolerant control system design," IEEE Transactions on Control Systems Technology, vol. 14, no. 2, pp. 284-292, 2006.

[7] L. Liu, Y. Shen, E. H. Dowell, and C. Zhu, "A general $H_{\infty}$ fault tolerant control and management for a linear system with actuator faults," Automatica, vol. 48, no. 8, pp. 1676-1682, 2012.

[8] H. H. Niemann, "A model-based approach to fault-tolerant control," International Journal of Applied Mathematics and Computer Science, vol. 22, no. 1, pp. 67-86, 2012.

[9] J. C. D. Silva, A. Saxena, E. Balaban, and K. Goebel, "A knowledge-based system approach for sensor fault modeling, detection and mitigation," Expert Systems with Applications, vol. 39, no. 12, pp. 10977-10989, 2012.

[10] M. Petković, M. R. Rapaić, Z. D. Jeličić, and A. Pisano, “On-line adaptive clustering for process monitoring and fault detection," Expert Systems with Applications, vol. 39, no. 11, pp. 10226-10235, 2012.

[11] Y. Xu, S. Tong, and Y. Li, "Adaptive fuzzy fault-tolerant control of static var compensator based on dynamic surface control technique," Nonlinear Dynamics, vol. 73, no. 3, pp. 2013-2023, 2013.

[12] S. J. Yoo, "Actuator fault detection and adaptive accommodation control of flexible-joint robots," IET Control Theory and Applications, vol. 6, no. 10, pp. 1497-1507, 2012.

[13] D. Rotondo, F. Nejjari, and V. Puig, "A virtual actuator and sensor approach for fault tolerant control of LPV systems," Journal of Process Control, vol. 24, no. 3, pp. 203-222, 2014.

[14] M. Sami and R. J. Patton, "Active fault tolerant control for nonlinear systems with simultaneous actuator and sensor faults," International Journal of Control, Automation and Systems, vol. 11, no. 6, pp. 1149-1161, 2013.

[15] J. Yuan, G. Liu, and B. Wu, "Power efficiency estimation-based health monitoring and fault detection of modular and reconfigurable robot," IEEE Transactions on Industrial Electronics, vol. 58, no. 10, pp. 4880-4887, 2011.

[16] S. Ahmad, H. Zhang, and G. Liu, "Distributed fault detection for modular and reconfigurable robots with joint torque sensing: a prediction error based approach," Mechatronics, vol. 23, no. 6, pp. 607-616, 2013.

[17] B. Zhao and Y. Li, "Local joint information based active fault tolerant control for reconfigurable manipulator," Nonlinear Dynamics, vol. 77, no. 3, pp. 859-876, 2014.

[18] Y. Li, Y. Liu, X. Liu, and Z. Peng, "Parameter identification and vibration control in modular manipulators," IEEE/ASME Transactions on Mechatronics, vol. 9, no. 4, pp. 700-705, 2004.

[19] T. Sun, Y. M. Song, Y. G. Li, and J. Zhang, "Workspace decomposition based dimensional synthesis of a novel hybrid reconfigurable robot," Journal of Mechanisms and Robotics, vol. 2, no. 3, Article ID 031009, 8 pages, 2010.

[20] M. Biglarbegian, W. W. Melek, and J. M. Mendel, "Design of novel interval type-2 fuzzy controllers for modular and reconfigurable robots: theory and experiments," IEEE Transactions on Industrial Electronics, vol. 58, no. 4, pp. 1371-1384, 2011.

[21] R. Muller, M. Esser, M. Jansen, and B. Corves, "Modular control system for reconfigurable robot applications," in Proceedings of the IEEE International Symposium on Assembly and Manufacturing, pp. 1-5, May 2011.

[22] W.-H. Zhu and T. Lamarche, "Modular robot manipulators based on virtual decomposition control," in Proceedings of the IEEE International Conference on Robotics and Automation (ICRA '07), pp. 2235-2240, Rome, Italy, April 2007. 
[23] S. Kirchoff and W. W. Melek, "A saturation-type robust controller for modular manipulators arms," Mechatronics, vol. 17, no. 4-5, pp. 175-190, 2007.

[24] Z. Li, Development and Control of a Modular and Reconfigurable Robot with Harmonic Drive Transmission System, University of Waterloo, Waterloo, Canada, 2007.

[25] B. Zhao and Y. Li, "Multisensor fault identification scheme based on decentralized sliding mode observers applied to reconfigurable manipulators," Mathematical Problems in Engineering, vol. 2013, Article ID 327916, 9 pages, 2013.

[26] R. N. Murray, Z. X. Li, and S. S. Sastry, A Mathematical Introduction to Robotic Manipulation, CRC Press, Boca Raton, Fla, USA, 1994

[27] M. Sami and R. J. Patton, "Active fault tolerant control for nonlinear systems with simultaneous actuator and sensor faults," International Journal of Control, Automation and Systems, vol. 11, no. 6, pp. 1149-1161, 2013.

[28] L. Bai, Z. Tian, and S. Shi, "Robust fault detection for a class of nonlinear time-delay systems," Journal of the Franklin Institute, vol. 344, no. 6, pp. 873-888, 2007. 


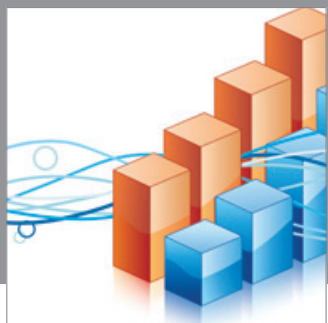

Advances in

Operations Research

mansans

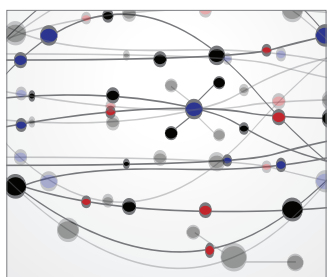

The Scientific World Journal
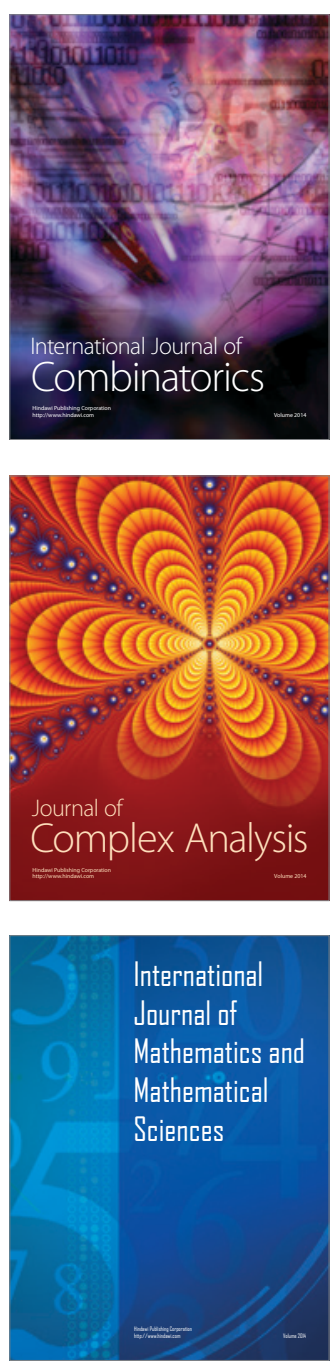
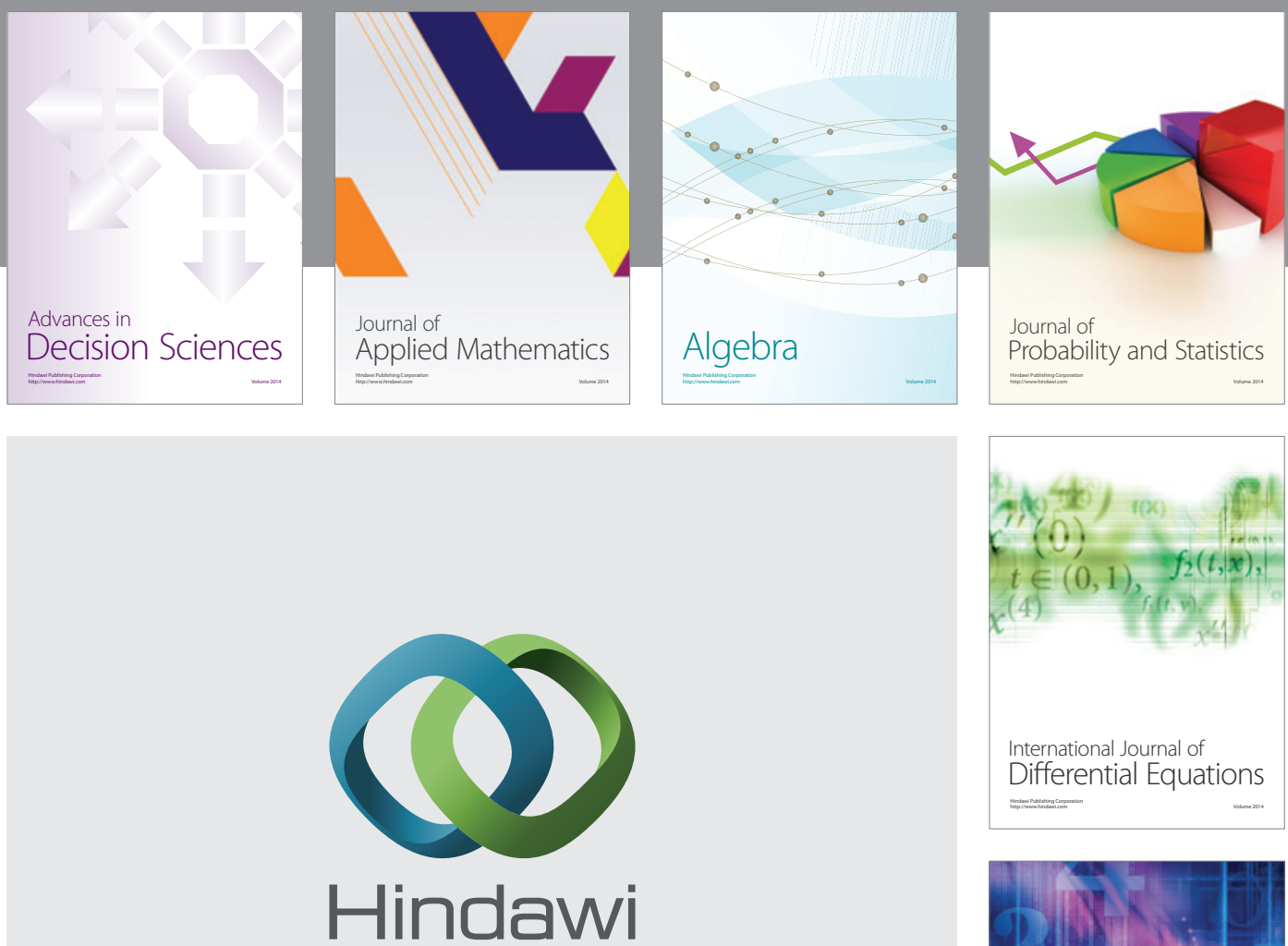

Submit your manuscripts at http://www.hindawi.com
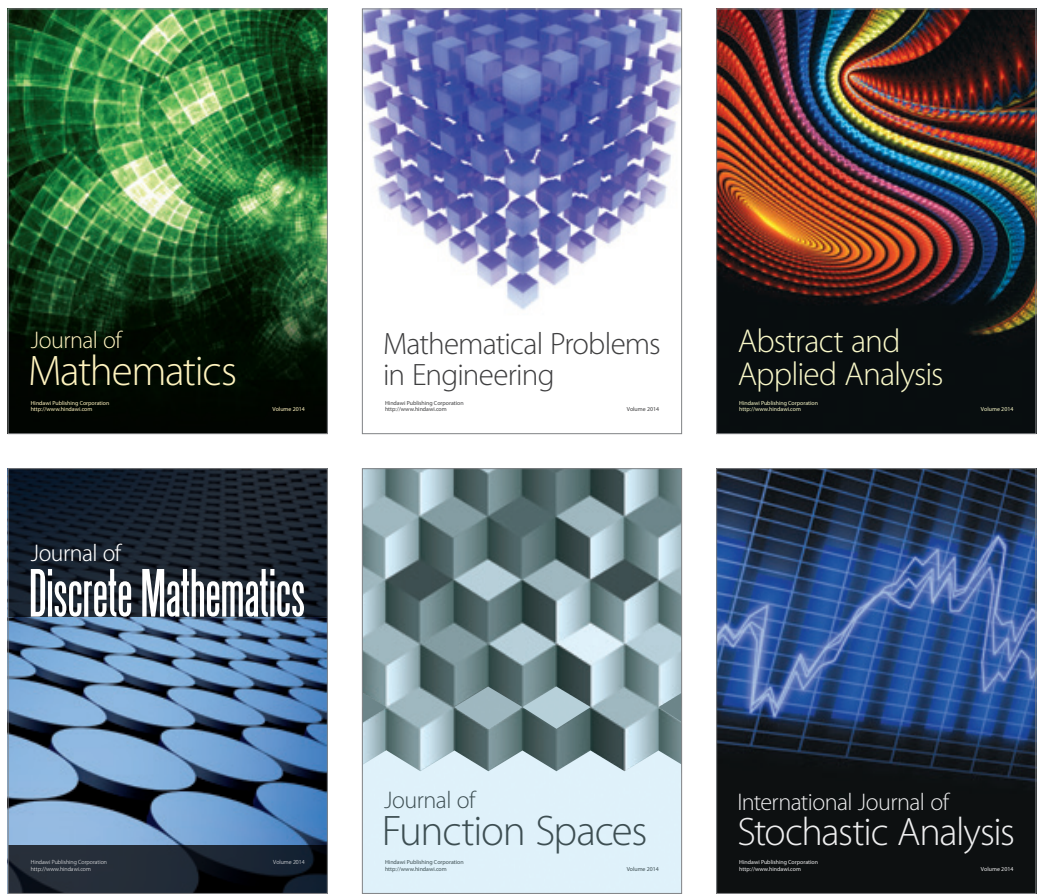

Journal of

Function Spaces

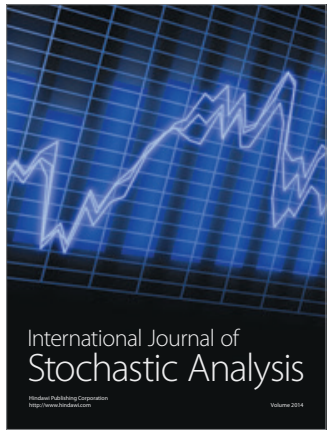

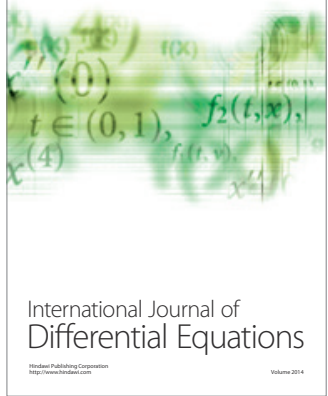
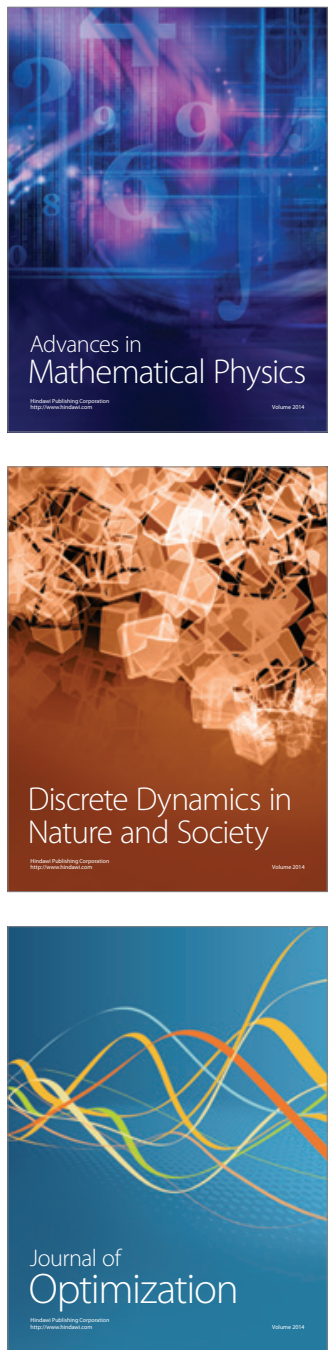